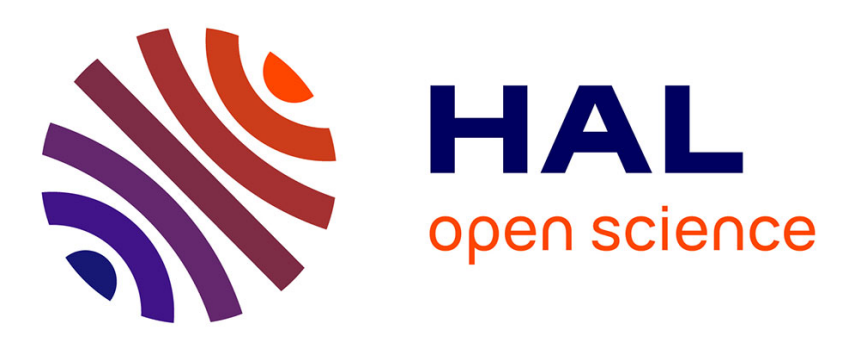

\title{
Large-Eddy simulation of an impinging heated jet for a small nozzle-to-plate distance and high Reynolds number Pierre Grenson, Hugues Deniau
}

\section{To cite this version:}

Pierre Grenson, Hugues Deniau. Large-Eddy simulation of an impinging heated jet for a small nozzleto-plate distance and high Reynolds number. International Journal of Heat and Fluid Flow, 2017, 68, pp.348-363. 10.1016/j.ijheatfluidflow.2017.09.014 . hal-01654589

\section{HAL Id: hal-01654589 \\ https://hal.science/hal-01654589}

Submitted on 6 Dec 2017

HAL is a multi-disciplinary open access archive for the deposit and dissemination of scientific research documents, whether they are published or not. The documents may come from teaching and research institutions in France or abroad, or from public or private research centers.
L'archive ouverte pluridisciplinaire HAL, est destinée au dépôt et à la diffusion de documents scientifiques de niveau recherche, publiés ou non, émanant des établissements d'enseignement et de recherche français ou étrangers, des laboratoires publics ou privés. 


\title{
Large-Eddy simulation of an impinging heated jet for a small nozzle-to-plate distance and high Reynolds number
}

\author{
Pierre Grenson*,a, Hugues Deniau ${ }^{\mathrm{b}}$ \\ a DAAA, ONERA, The French Aerospace Lab, 92190 Meudon, France \\ b DMPE, ONERA, The French Aerospace Lab, 31000 Toulouse, France
}

\section{A R T I C L E I N F O}

\section{Keywords:}

Jet impingement

Heat transfer

Large-eddy simulation

Turbulent flow

Primary structure

Hot spot

\begin{abstract}
A B S T R A C T
This paper reports on the investigation of an original impinging jet configuration through a wall-resolved largeeddy simulation. The heated jet issues from a fully developed pipe flow at temperature of $130{ }^{\circ} \mathrm{C}$ and a Reynolds number based on the bulk velocity of 60000 . The impinged plate is located three diameters downstream of the pipe exit. The CFD results have been validated against a specifically-created experimental database (Grenson et al., 2016). The overall statistical fields are well retrieved by the simulation both in the free jet and the wall jet region. In particular, the secondary maximum at the radial location $r / D=2$ in the Nusselt number distribution is well predicted by the simulation. The underlying mechanisms from which the secondary maximum originates has been investigated. This analysis revealed that small-scale hot spots of strong convective heat transfer coefficient are responsible for the emergence of this secondary maximum. It is shown that the hot spots can be associated either to local unsteady "separation" of the flow or streaks-like structures above the impinging plate.
\end{abstract}

\section{Introduction}

The impinging jet configuration is of widespread use in industrial applications (e.g. turbine blade cooling, aircraft leading edge heating) due to the high heat transfer rate experienced by the impinged surface (Han and Goldstein, 2001). It also remains a relevant configuration for turbulence modeling and numerical simulation assessment (Zuckerman and Lior, 2006; Dewan et al., 2012) due to the variety of the flow regions that coexist (Fig. 1). In this framework, an Onera joint project has been dedicated to a particular jet impingement configuration, which was selected for its high Reynolds number $\operatorname{Re}_{D}=60000$, small nozzle-toplate distance $H / D=3$ and temperature difference between the heated jet $\left(T_{j}=130{ }^{\circ} \mathrm{C}\right)$ and the ambient atmosphere $\left(T_{e}=25^{\circ} \mathrm{C}\right)$. Because this particular configuration had never been investigated in the past, an exhaustive experimental database has been specifically created for numerical validation purposes by Grenson et al. (2016). The goal of the present paper is to numerically investigate the flow field and heat transfer distribution of this configuration through a wall-resolved largeeddy simulation (LES).

In the last decade, several LES were carried out on impinging jet configurations. Most of these simulations (Hadžiabdić and Hanjalić, 2008; Uddin et al., 2013; Aillaud et al., 2016; Natarajan et al., 2016) dealt with a turbulent jet issuing a fully developed pipe at Reynolds number $\operatorname{Re}_{D} \sim 20000$ and for a nozzle-to-plate distance $H / D=2$.
Lodato et al. (2009) performed a LES for the same nozzle-to-plate distance but a higher Reynolds number $\operatorname{Re}_{D}=70000$. Unfortunately, the used grid was to coarse to meet the requirements of a wall-resolved LES and no information about the heat transfer distribution was reported. It should be noticed that the aforementioned works were dedicated to isothermal jet configuration, for which the jet temperature $T_{j}$ is identical to the ambient temperature $T_{e}$.

To the authors knowledge, the present study is the first wall-resolved LES of an impinging jet configuration at such a high Reynolds number and for a heated jet.

Since the measurements of Gardon and Akfirat (1965) and Baughn and Shimizu (1989), the Nusselt number has been shown to feature a double-peak shaped distribution for impinging configurations with a nozzle-to-plate distance less than $H / D=4$. The first peak is located at the stagnation point while the secondary peak is generally located around the radial position $r / D=2$. Its intensity increases with the jet Reynolds number (Lee and Lee, 1999). Various explanations about the mechanisms behind this non-monotonic distribution have been proposed, generally on the basis of unsteady numerical simulations. Hadžiabdić and Hanjalić (2008) related the secondary maximum to the reattachment of local unsteady separations of the flow, by taking up the arguments of Popiel and Trass (1991) who observed such separations experimentally. The unsteady separation in impinging flows was first characterised by Didden and Ho (1985). It results from the

\footnotetext{
* Corresponding author.

E-mail address: pierre.grenson@onera.fr (P. Grenson).
} 


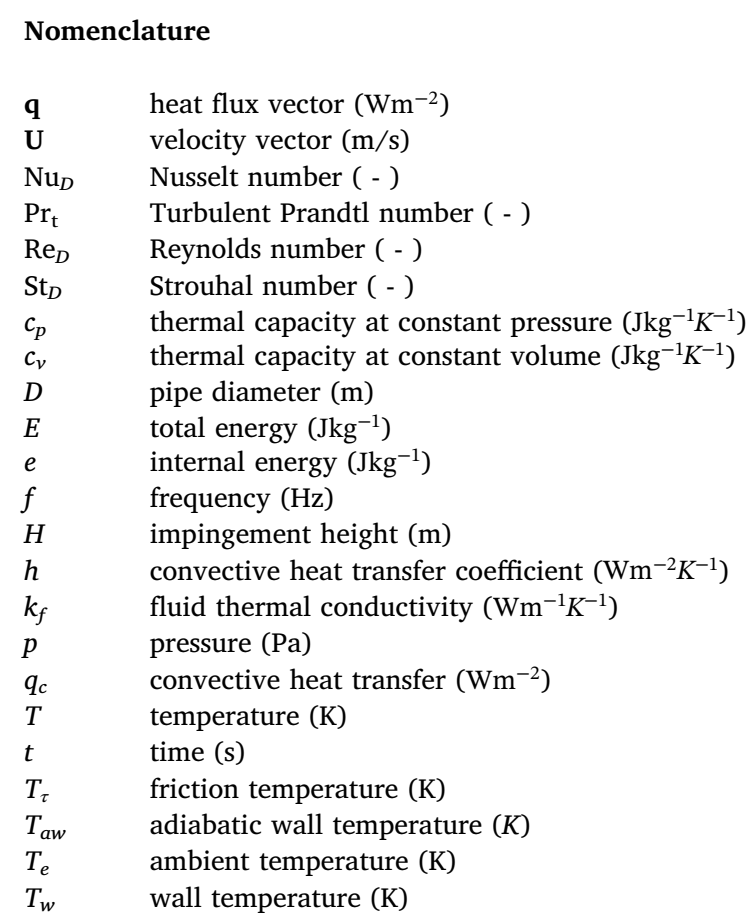

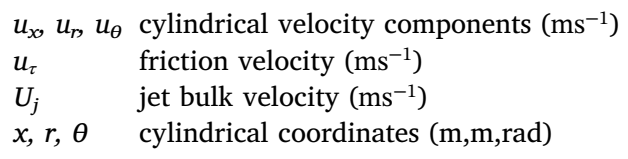

Greek symbols

$\begin{array}{ll}\Delta t & \text { time step (s) } \\ \Delta & \text { spatial discretisation (m) } \\ \delta_{\omega} & \text { vorticity thickness (m) } \\ \eta & \text { effectiveness }(-) \\ \mu & \text { molecular viscosity }\left(\mathrm{kgm}^{-1} \mathrm{~s}^{-1}\right) \\ \nu & \text { kinematic viscosity }\left(\mathrm{m}^{2} \mathrm{~s}^{-1}\right) \\ \rho & \text { density }\left(\mathrm{kgm}^{-3}\right) \\ \tau & \text { viscous stress tensor }\left(\mathrm{Nm}^{-2}\right) \\ \tau_{c} & \text { characteristic time }(\mathrm{s}) \\ \tau_{w} & \text { wall friction }\left(\mathrm{Nm}^{-2}\right) \\ & \\ \text { Operators } & \\ & \\ <> & \text { time-averaged quantity } \\ <>\text { az. } & \text { azimuthally-averaged quantity } \\ + & \text { wall unit } \\ \text { RMS } & \text { root-mean-square }\end{array}$

$\Delta \quad$ spatial discretisation (m)

$\delta_{\omega} \quad$ vorticity thickness $(\mathrm{m})$

$\eta \quad$ effectiveness ( - )

$\mu \quad$ molecular viscosity $\left(\mathrm{kgm}^{-1} \mathrm{~s}^{-1}\right)$

$\nu \quad$ kinematic viscosity $\left(\mathrm{m}^{2} \mathrm{~s}^{-1}\right)$

$\rho \quad$ density $\left(\mathrm{kgm}^{-3}\right)$

$\tau \quad$ viscous stress tensor $\left(\mathrm{Nm}^{-2}\right)$

$\tau_{c} \quad$ characteristic time (s)

$\tau_{w} \quad$ wall friction $\left(\mathrm{Nm}^{-2}\right)$

RMS root-mean-square interaction of the large-scale vortices generated in the free jet, often referred to as primary structures, with the plate. Flow separation is promoted by the adverse pressure gradient encountered in the vicinity these primary structures. This process eventually leads to the emergence of counter-rotating vortices, so-called secondary structures, which are advected along the plate as the primary structure progresses downstream. On the basis of the Reynolds analogy between heat and momentum transfer, Popiel and Trass (1991) and Hadžiabdić and Hanjalić (2008) related the dip between the two Nusselt number peaks to the heat transfer decrease induced by this kind of flow separation.

In their large-eddy simulation, Uddin et al. (2013) did not observe any unsteady separation but noticed the emergence of small spots of high convective heat transfer coefficient, referred as "hot spots", in the region of the secondary maximum. Those spots are convected as the primary structure travels along the plate. By means of a direct numerical simulation of an impinging jet at $\operatorname{Re}_{D}=10000$ and $H / D=2$,

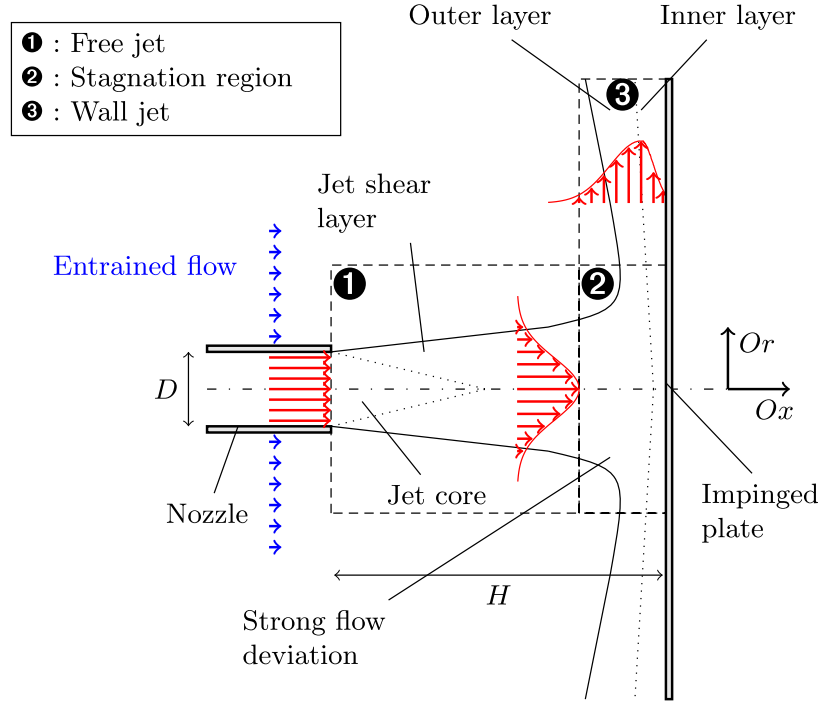

Fig. 1. Schematic description of a round jet impinging on a flat plate.
Dairay et al. (2015) showed that the hot spots are related to the instability of the secondary structures (unsteady separation). Thanks to an analysis of higher-order statistics from their LES, Aillaud et al. (2016) highlighted that the most probable event in the vicinity of the secondary maximum location is a cold fluid flux towards the plate, which leads to heat flux enhancement.

The purpose of the present work is to highlight which mechanisms take place for a high Reynolds number impinging jet which exhibits a well-pronounced secondary maximum.

The present paper is organized as follows. First, the computational setup is presented (Section 2). Next the simulation results are validated against the experimental database (Section 3). Finally, the full 3D timeresolved data from the simulation are analysed to get insight into the mechanisms related to the heat transfer distribution on the plate (Section 4).

\section{Computational setup}

The computational domain, sketched in Fig. 2(a), models the experimental configuration. It consists of a cylindrical domain connected to a circular pipe of diameter $D$. The impinged plate is represented by a disk of diameter $12 D$ located $3 D$ downstream of the pipe exit. Due to its large external diameter of $2.25 \mathrm{D}$, the complete nozzle geometry is also taken into account. The ambient domain is included up to $3 D$ upstream of the pipe exit. The origin of the orthogonal coordinate system is located at the pipe outlet center. The jet axis is along the $x$ direction and is taken as positive in the jet mean direction. The radial direction $r$ is oriented along the plate.

\subsection{Governing equations}

The flow dynamics is governed by the compressible Navier-Stokes equations, expressed in their conservative form herebelow:

$\frac{\partial \rho}{\partial t}+\operatorname{div}(\rho \mathbf{U})=0$

$\frac{\partial(\rho \mathbf{U})}{\partial t}+\operatorname{div}(\rho \mathbf{U} \otimes \mathbf{U}+p \mathbf{I}-\tau)=0$ 


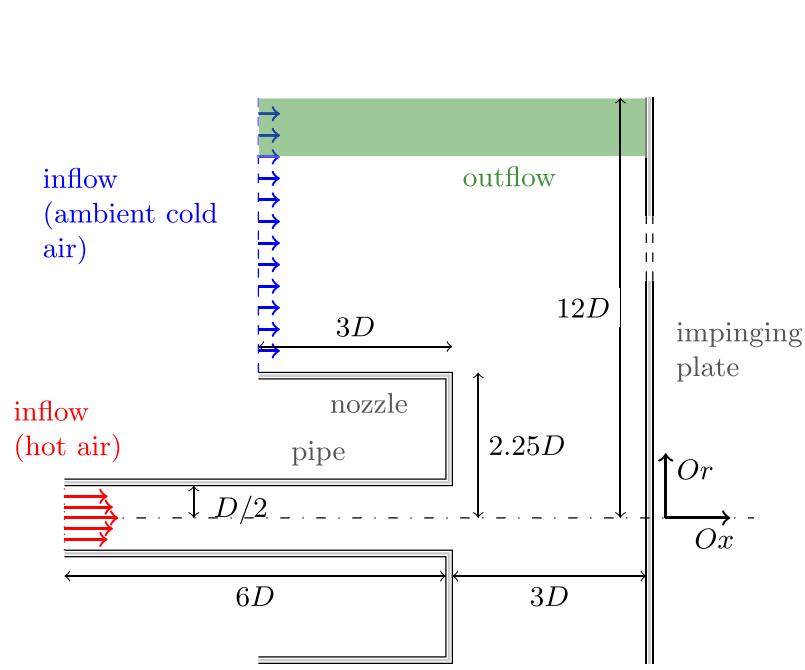

(a) Computational domain and boundary conditions.

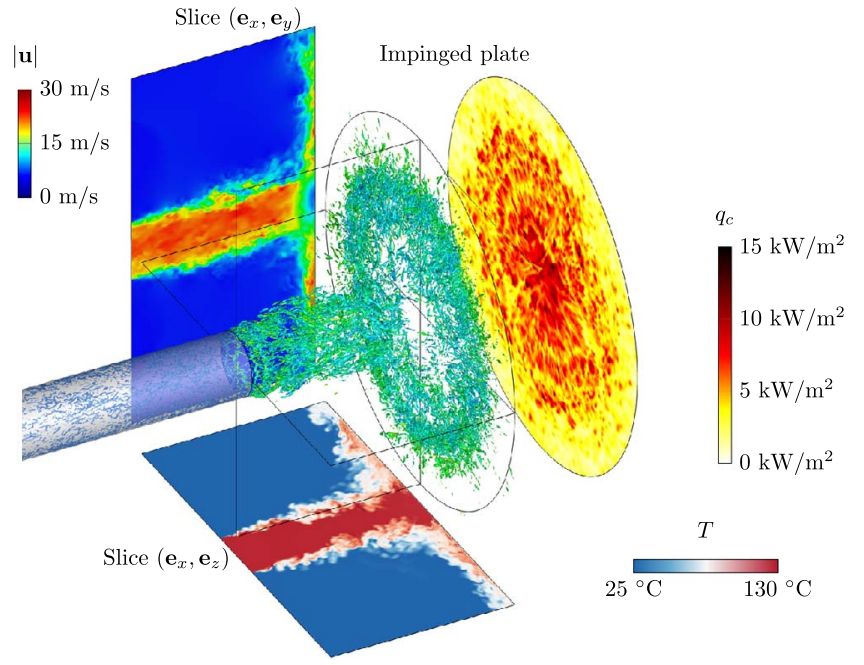

(b) Instantaneous results from the simulation. (Complete nozzle geometry is not represented).

Fig. 2. Computational domain.

$$
\frac{\partial(\rho E)}{\partial t}+\operatorname{div}(\rho E \mathbf{U}+p \mathbf{U}-\tau \cdot \mathbf{U}+\mathbf{q})=0
$$

They correspond respectively to the conservation of mass, momentum $\rho \mathbf{U}$ and total energy $\rho E$ per unit volume, $E$ being equal to the sum of the internal energy $e$ and kinetic energy $\frac{1}{2} \mathbf{U}^{2}$. Considering that air is a Newtonian fluid and obeys the Stokes hypothesis, the stress tensor $\tau$ is related to the velocity field through the molecular viscosity $\mu$ :

$\tau=\mu(T)\left[\operatorname{grad} \mathbf{U}+\operatorname{grad}^{t} \mathbf{U}-\frac{2}{3}(\operatorname{div} \mathbf{U}) \mathbf{I}\right]$

The molecular viscosity $\mu$ dependence to the temperature $T$ is modeled by the Sutherland's law ( $\mu$ varies up to $35 \%$ in the temperature range considered in this study).

The heat flux due to conduction in the fluid $\mathbf{q}$ is modeled by the Fourier law:

$\mathbf{q}=-k_{f}(T) \operatorname{grad} T$

where $k_{f}$ is the thermal conduction coefficient which is computed from the molecular viscosity by means of the Prandtl number:

$\operatorname{Pr}=\frac{\mu(T) c_{p}}{k_{f}(T)}$

which can be considered constant and equal to 0.71. Regarding the jet temperature of the present work, the fluid can be considered as a calorically perfect gas, for which $\gamma=c_{p} / c_{v}=1.4$. The system (1)-(3) is then closed by the following equations:

$\frac{p}{\rho}=\mathscr{R} T$

$e=c_{v} T$

where $\mathscr{R}=287 \mathrm{Jkg}^{-1} \mathrm{~K}^{-1}$ and $c_{v}=717 \mathrm{Jkg}^{-1} \mathrm{~K}^{-1}$.

The large-eddy simulation approach consists in resolving only the largest scales of turbulent structures in the flow. In this study, LES is performed by means of an explicit spatial filtering of the five conservative variables at the end of each time iteration. That explains why no explicit subgrid-scale (SGS) terms are included in both the momentum and energy equations (2) and (3). This approach, often referred to as relaxation filtering, has been proven accurate for various free jet flow simulation by Bogey and Bailly (2006).

\subsection{Numerical simulation procedure}

The governing equations (1)-(3) are numerically solved on a structured mesh using the in-house finite-volume solverelsA (french acronym for "Aerodynamic Simulation Software Package") (Cambier et al., 2013). The spatial discretisation is performed with the 6th-order compact finite-volume scheme of Fosso et al. (2010) that allows for the accurate resolution of turbulent structures which span at least 6 cells (compared to the 20 cells generally recommended for a classical second-order Roe upwind scheme (Sagaut et al., 2013)). The stability of the computations is enforced using the 8th-order compact filter of Visbal and Gaitonde (2002), with the filter parameter $\alpha_{f}=0.47$. This filter plays also the role of the LES explicit filter, by dissipating the energy of the underesolved turbulent structures.

Time integration is performed by the explicit 4-step scheme of Runge-Kutta. The dimensionless time step $\Delta t U_{j} / D=3 \times 10^{-5}$ is chosen for stability reasons so that the acoustic CFL number remains less than 1.4 in the smallest cell of the domain.

\subsection{Meshing strategy}

The mesh has been designed to respect the commonly acknowledged guidelines for a large-eddy simulation (Sagaut et al., 2013). In the free-jet region, the cell size is based on the vorticity thickness $\delta_{\omega}=\frac{U_{j}}{\partial\left\langle u_{x}\right\rangle / \partial r}$ of the jet. It does not excess $\Delta x=\delta_{\omega} / 10, \Delta r=\delta_{\omega} / 45$ and $r \Delta \theta=\delta_{\omega} / 5$. In the wall-jet region, the meshing strategy enables to meet the wall-resolved constraints of Piomelli and Chasnov (1996) based on the viscous length $l_{\nu}=u_{\tau} / \nu$. The cell size in each direction are smaller than $\Delta x^{+}=\Delta x / l_{\nu}=2, \Delta r^{+}=\Delta r / l_{\nu}=25$ and $(r \Delta \theta)^{+}=(r \Delta \theta) / l_{\nu}=25$ in the whole domain of interest. The final mesh, after several design iterations, contains $350 \times 10^{6}$ cells, including $100 \times 10^{6}$ cells in the pipe only. This mesh size is one order magnitude higher than in the previous LES (Aillaud et al., 2016; Lodato et al., 2009; Hadžiabdić and Hanjalić, 2008; Uddin et al., 2013), dedicated to lower Reynolds number jet $\left(\operatorname{Re}_{D} \sim 20000\right)$.

The meshing strategy is illustrated in Fig. 3, which shows the blocks and the number of cells along the edges in Oxy and Oyz plane. As on shown in Fig. 3(a), a particular block topology, so-called "O-4H", was implemented in order to maintain the size of the cell in the circumferential direction small enough to meet the requirement on $(r \Delta \theta)^{+}$. 8 blocks (blue blocks in Fig. 3 ) have been added around a central O-grid (yellow blocks in Fig. 3), associated with the circular pipe and the jet 


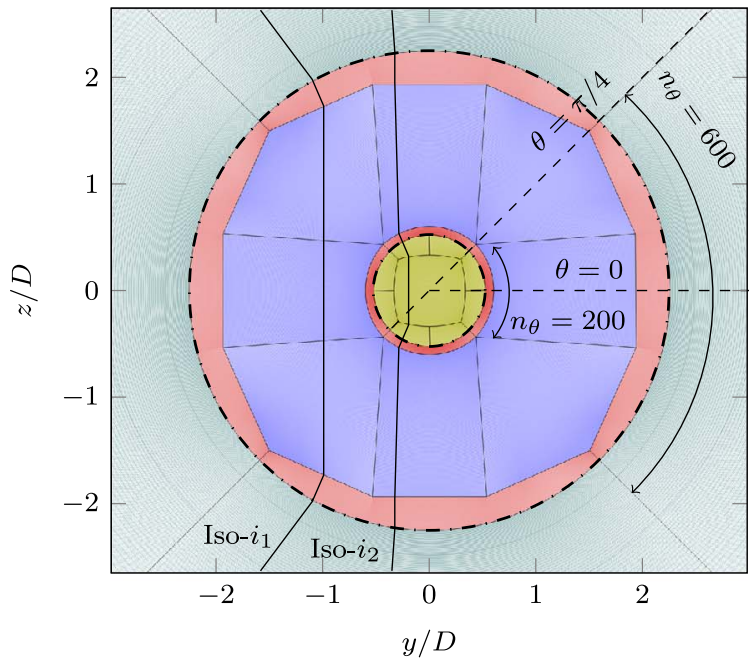

(a) Oyx plane.

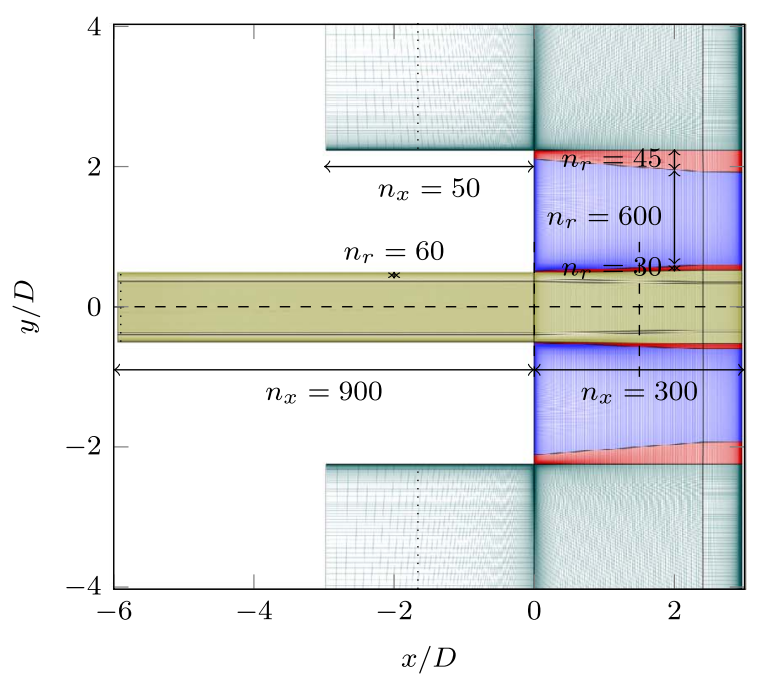

(b) Oxy plane.

Fig. 3. Mesh topology. (Yellow) Pipe and jet core blocks. (Blue) O-4H blocks. (Red) Connecting blocks. (Green) Outer blocks.

core. This topology allows to concentrate meshing lines issuing from the outer part of the computational domain into the region of interest above the impinged plate without propagating them throughout the central O-grid (as illustrated by the meshing line $i_{1}$ in Fig. 3)(a). Traditional O-grid for the whole plate would have led to unnecessary small cells in that region that, in turn, would cause severe limitation of the maximum allowable time step.

\subsection{Boundary conditions}

At the pipe inflow, the mean velocity profile corresponding to the fully developed pipe is prescribed. The jet bulk velocity $U_{j}$ is $25.7 \mathrm{~m} / \mathrm{s}$. The pipe inflow temperature is set uniform and constant at the jet temperature $T_{j}=130^{\circ} \mathrm{C}$. Because the pipe is fully developed, one needs to generate resolved flow fluctuations upstream of the pipe exit. For that purpose two types of velocity perturbations are jointly employed (see Fig. 4):

1. Vortex rings (Bogey et al., 2003) are generated in the flow field in the close vicinity of the pipe wall. Their effect on the flow field is similar to that of a small geometrical step located at the same position on the wall. This allows to trip the boundary layer which rapidly evolves into a turbulent state.

2. Isotropic homogeneous turbulence, based on the approach of Bechara et al. (1994), is injected over the whole inflow section in order to feed the core region of the pipe with resolved turbulent content.

Preliminary tests have shown that a pipe length of 6 diameters was necessary for these perturbations to transform into a realistic developed flow of the whole pipe section. As shown in Fig. 5, both the mean velocity profile and the complete Reynolds tensor at the pipe exit $(x=0)$ are in fair agreement with the measurements.

At the external inflow, which corresponds to the ambient cold air entrained by the jet, the mean velocity and a uniform temperature $T_{e}=25{ }^{\circ} \mathrm{C}$ profiles are imposed. The velocity profiles at this location are available from our experiments (Fig. 6).

The no-slip boundary condition is applied to the walls. Both the pipe and the nozzle are taken adiabatic. The impinged plate is considered isothermal, by imposing a uniform temperature of $T_{w}=25^{\circ} \mathrm{C}$. Regarding the experiments, this thermal configuration corresponds to the instant at which the plate, initially at ambient temperature, is suddenly submitted to the hot flow. At this time, the temperature difference between the jet and the plate is the highest, that is the best configuration for accurate heat flux $q_{c}$ prediction. Convective heat exchanges are generally quantified by the convective heat transfer coefficient defined as:

$h=\frac{q_{c}}{T_{w}-T_{a w}}$

Contrary to the configurations dedicated to isothermal jets, the adiabatic wall temperature $T_{a w}$ is not equal to the jet temperature $T_{j}$ for our nonisothermal case, because of the mixing between hot and cold fluid. In order to determine this a priori unknown temperature, an additional simulation (referred to as LES-ADIA in the following) has been performed with an adiabatic boundary condition $\left(q_{c}=0\right)$ prescribed on the plate.

The outflow boundary condition consists of a non-reflective boundary condition (Tam and Dong, 1996) applied at $r / D=12$. A sponge layer relaxes the pressure to the ambient pressure $p_{e}=101325 \mathrm{~Pa}$ and dissipates the vortical structures convected from the wall jet.
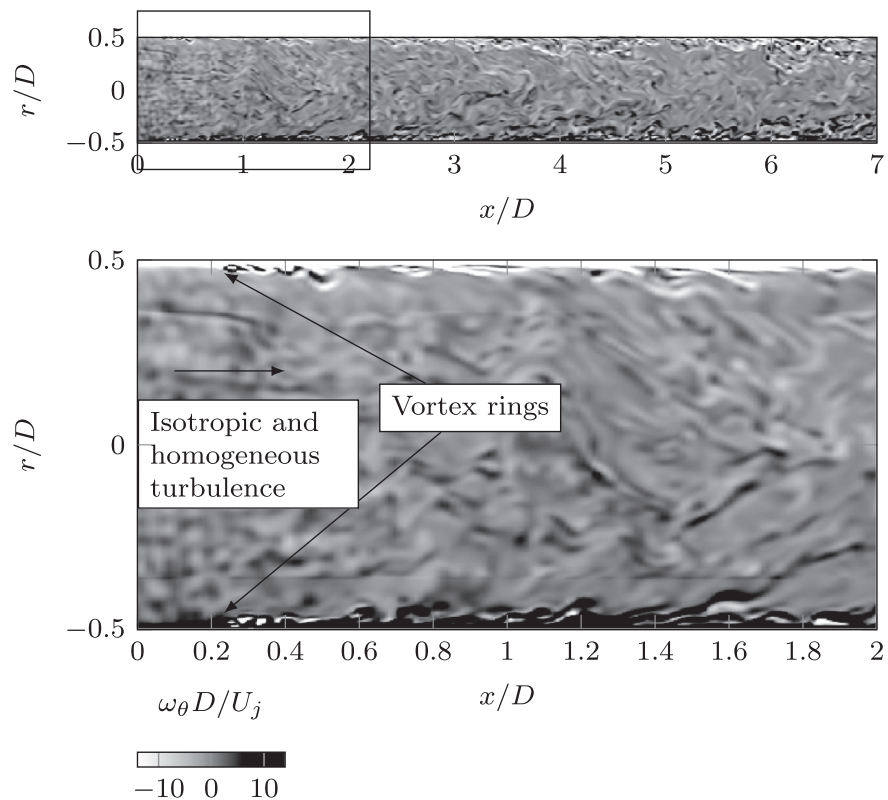

Fig. 4. Illustration of the strategy for generating a fully-developed pipe flow. Azimuthal vorticity $\omega_{\theta}$ in a cut plane of the circular pipe from a preliminary simulation of an isolated pipe of length $7 D$. 

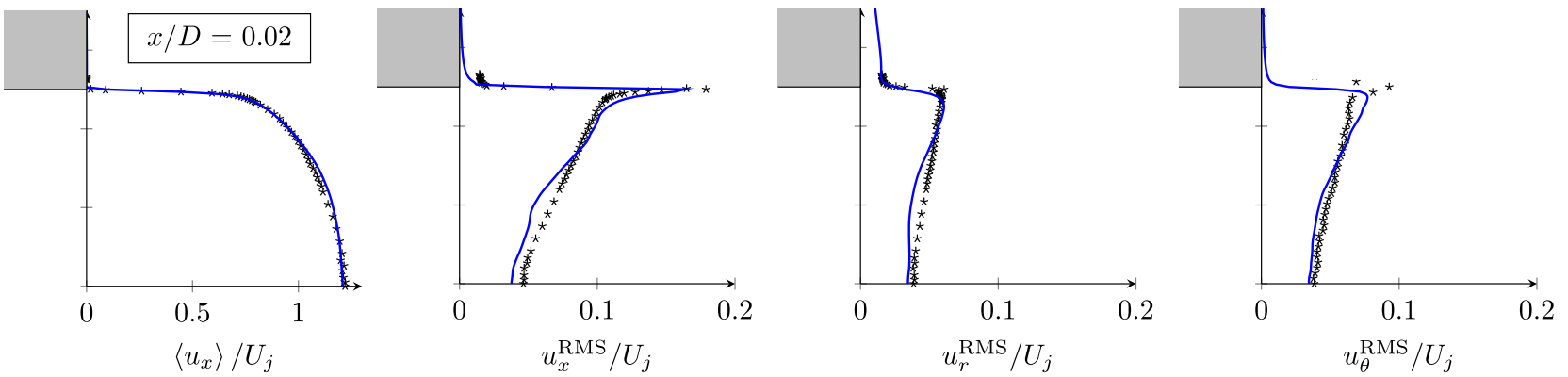

Fig. 5. Pipe outlet conditions at $x / D=0.02$. Mean and fluctuating profiles of the velocity field. ( $\star$ ) Experimental data. ( $\longrightarrow$ ) LES.

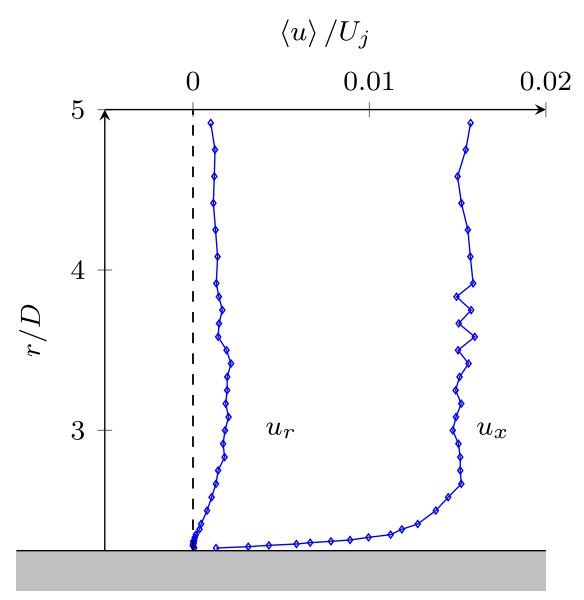

Fig. 6. Axial and radial velocity profiles imposed at the external inflow boundary condition $(x=-3 D)$ from the measurements.

\subsection{Simulation duration}

The total physical simulated time was 21 cycles, where one cycle has a duration $\tau_{c}=22.5 \mathrm{D} / U_{j}$, which corresponds to the period associated with the column mode $\mathrm{St}_{D}=f D / U_{j}=0.4$ of the free jet. Once the transient has been evacuated (after 8 cycles), the statistical fields are obtained through both time and azimuthal averaging of the instantaneous flow fields. It has been observed that both the mean and fluctuating fields are fully converged after 8 cycles.

\subsection{Data extraction}

In the scope of physical analysis, instantaneous fields of both velocity and temperature have also been extracted at a frequency of $14 \mathrm{kHz}$, as well as instantaneous distribution of the convective heat flux $q_{c}$ and friction vector $\overrightarrow{\tau_{w}}$ on the impinged plate. Velocity and temperature signals have been recorded at a thousand of probes spread in the domain of interest, at a sampling frequency of $550 \mathrm{kHz}$.

Fig. 2(b) shows an instantaneous isosurface of $Q$-criterion along with $O x y$ and $O x z$ slices of both velocity and temperature fields. The instantaneous heat transfer distribution on the impinged plate is also represented. It shows that the flow exhibits a large range of turbulent scales, as expected for a high Reynolds number jet which exits a fully turbulent pipe.

\section{Simulation validation}

\subsection{Statistical fields}

\subsubsection{Dynamical and thermal flow fields}

The mean and fluctuating velocity fields from the experiments and the LES are compared in Fig. 7. The agreement is satisfactory for the mean flow (Fig. 7, center). The major flow features are well retrieved: the radial expansion of the annular shear layer in the free-jet region, the flow deviation in the stagnation region with zero velocity at the center of the impinged plate and the velocity decrease in the wall-jet region. The highest radial velocity $\left\langle u_{r}\right\rangle / U_{j} \simeq 1.1$ is reached at the radial location $r / D=1$, which generally defines the limit between the stagnation and the wall-jet region. The entrainements effects, such as the recirculation bubble behind the nozzle external case, are also correctly captured, as highlighted by the streamlines (Fig. 7, left).

The field of the velocity fluctuations is satisfactorily reproduced by the simulation (Fig. 7, right). The free-jet region is characterized by high levels of turbulent kinetic energy $k$ in the shear layer while the stagnation region features damping of the velocity fluctuations due to the wall blocking effect. In the wall jet, the turbulent kinetic energy progressively increases to reach its highest level around the radial location $r / D=1.7$ (loupe in Fig. 7, right). Although those features are well captured by the present simulation, some discrepancies should however be mentioned. It notably appears that fluctuations are overestimated in the free-jet shear layer and this from the very beginning of the jet (Fig. 7, right). Fig. 8 shows that the kinetic energy fluctuations primarily comes from the overestimation of the radial velocity component, especially between $r / D=0.5$ and 1.0. This overestimation must be linked to the slight over-prediction of the shear layer thickness as evidenced by the axial mean velocity profiles and the temperature profiles gathered in Fig. 8. This Figure also shows that temperature fluctuations are strongly overestimated at the beginning of the free jet. Those differences between the LES data and experiments are not due to the pipe exit conditions, which are fairly reproduced. Furthermore, a mesh convergence study (not shown here) revealed that the mean and fluctuating field prediction in the free-jet region are not improved on a refined mesh for which the number of cells in the axial direction has been doubled. The influence the SGS modeling have also been assessed, with no influence on the predicted fields with a WALE approach (Nicoud and Ducros, 1999). We finally attribute the fluctuation overestimation to the presence of a spurious pressure wave that have been observed traveling back and forth in the $6 D$-pipe. This phenomenon has a frequency $S_{D} \simeq 0.8$ close to the first harmonic of the free-jet column mode and could therefore sightly excites the shear layer.

Because the heat transfer is principally linked to the flow dynamics in the wall jet region, a detailed validation is performed for both velocity and temperature profiles at three different locations along the plate (Fig. 7)(a). In order to assess the influence of the thermal boundary condition, the statistical fields from both the LES and LESADIA simulations are represented. The agreement on the mean radial velocity is excellent (Fig. 9)(a) with the thickening of the very thin boundary layer (inner layer) and the expansion of the outer shear layer. This figure also shows that the thermal condition of the plate does not influence the velocity profiles. This is not the case for the mean temperature (Fig. 9)(b) which exhibits very different profiles in the vicinity of the wall. The profiles associated with the adiabatic boundary condition (LES-ADIA) are the closest to the measurements. Indeed, the temperature profiles were measured at equilibrium condition. At this steady-state, the convective heat flux is low: it balances the heat 

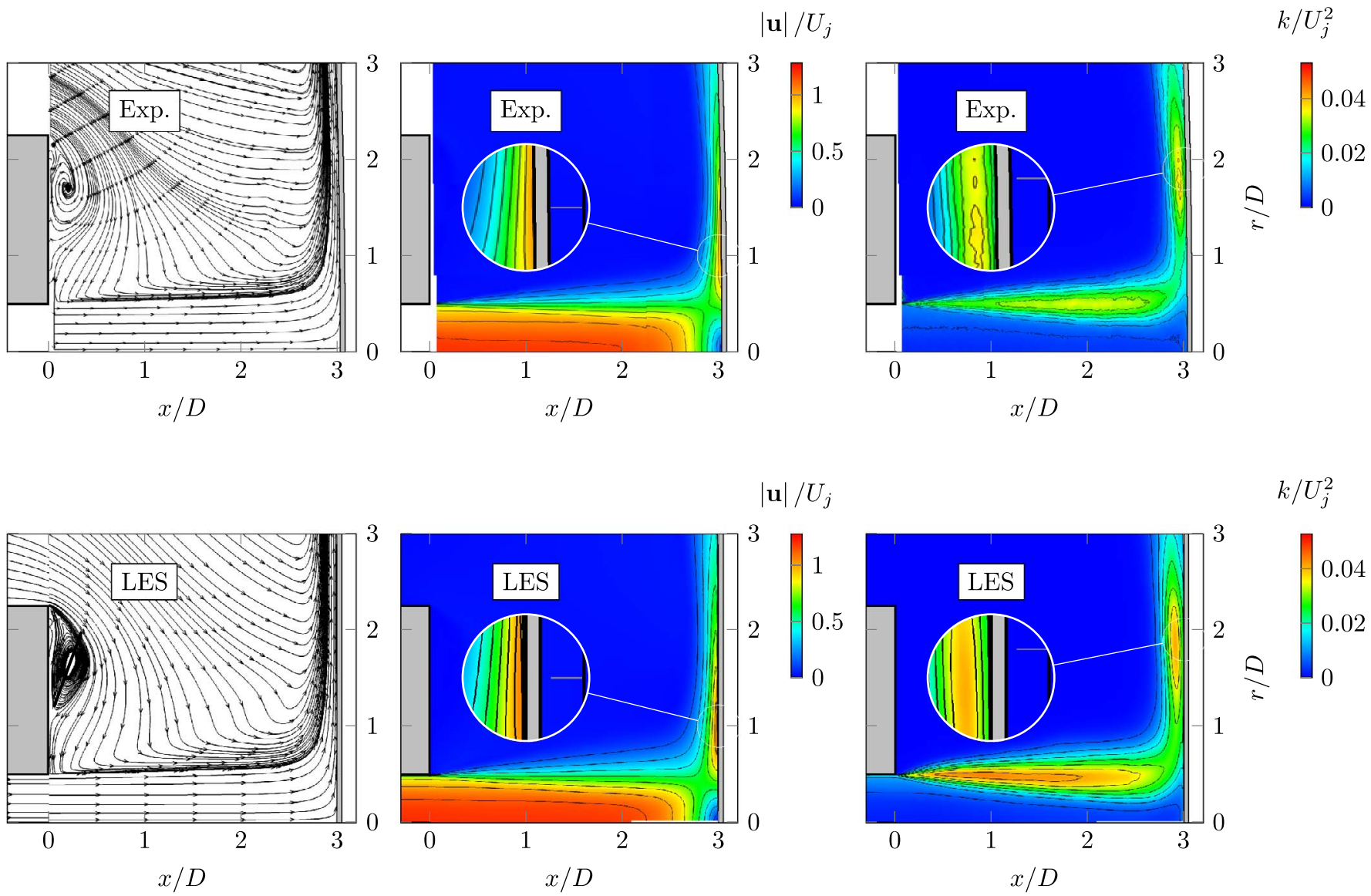

Fig. 7. Mean and fluctuating velocity fields. (Top) Experimental data. (Bottom) LES. (Left) Streamlines. (Center) Velocity magnitude. (Right) Turbulent kinetic energy $k$.

diffusion in the thin plate and the low losses on the rear face (more details are provided in Grenson et al. (2016)).

Fig. 9(c) and (d) show that the axial and radial velocity fluctuations are insensitive to the thermal boundary condition. In addition, they are accurately reproduced by the simulation for all profiles excepted at the radial location $r / D=1$ where the fluctuations of the radial velocity in the near-wall region are overestimated by $20 \%$. The origin of this difference, which has also been observed in previous LES of the literature (Uddin et al., 2013; Aillaud et al., 2016), is still unclear. Two explanations could be proposed. This discrepancy might simply be a consequence of the overestimation of the fluctuations observed in the free-jet region (Fig. 8). Alternatively one could incriminate the explicit filtering strategy which only dissipates energy of the undiscretised scales and is therefore not able to take into account energy backscatter. Lodato et al. (2009) indeed showed that the use of an advanced SGS model (namely, a wall-adapting mixed similarity model), designed to model the stress tensor anisotropy and the energy backscatter, gives better results for the fluctuations in this region.

The temperature fluctuations are represented in Fig. 9(e). Contrary to the velocity field, the thermal boundary condition alters the temperature profiles, especially in the near-wall region. The temperature fluctuations for the isothermal condition exhibit a small peak in the inner boundary layer, as highlighted by the loupes in Fig. 9(e). In the case of the adiabatic condition, they feature only a change in their slope. This behaviour is closer to the measurements. Fig. 9(e) also shows that the temperature fluctuations from both LES and LES-ADIA are overestimated compared to the experimental data. This is certainly a consequence of the mixing overestimation in the free-jet shear layer that we mentioned herebefore.

These results indicate that the thermal coupling between the flow and the impinged plate is weak. In other words, the thermal effects do not influence the flow dynamics. It should then be expected that the convective heat flux coefficient is entirely determined by the flow velocity and is independent of the thermal boundary condition, at least for wall temperature comprised between $T_{w}=25^{\circ} \mathrm{C}$ and $T_{a w}$.

\subsubsection{Inner layer profiles}

In order to assess the degree of universality of the inner boundary layer, both velocity and temperature profiles form the LES with the isothermal wall condition have been scaled in wall unit. The mean radial velocity component $u_{r}$ is scaled by the friction velocity $u_{\tau}=\sqrt{\tau_{w} / \rho}$ to form the non-dimensional velocity $U^{+}=u_{r} / u_{\tau}$. The mean temperature is scaled according to the friction temperature $T_{\tau}$, defined as:

$T_{\tau}=\frac{q_{c}}{\rho c_{p} u_{\tau}}$,

to form the non-dimensional temperature:

$\Theta^{+}=\frac{T-T_{w}}{T_{\tau}}$,

Both friction velocity and friction temperature vary along the impinged plate. Non-dimensional profiles of $U^{+}$are plotted in Fig. 10(a) against the distance to the wall, also expressed in wall unit $(H-x)^{+}$, for different radial locations. In Fig. 10(b), non-dimensional profiles of $\Theta^{+}$are plotted against $(H-x)^{+}$multiplied by the Prandtl number Pr, which is the non-dimensional parameter that relates thermal diffusion to viscous diffusion. It is seen from these Figures that both velocity and temperature profiles collapse to the universal linear law $\left(U^{+}=(H-x)^{+}\right.$ and $\left.\Theta^{+}=\operatorname{Pr} \cdot(H-x)^{+}\right)$in the viscous sublayer, which extends up to $(H-x)^{+} \simeq 10$, as already observed by Hattori and Nagano (2004) from a direct numerical simulation of a $\mathrm{Re}_{D}=10000$ plane impinging jet. Beyond that distance, the overall profile similarity is lost except for 


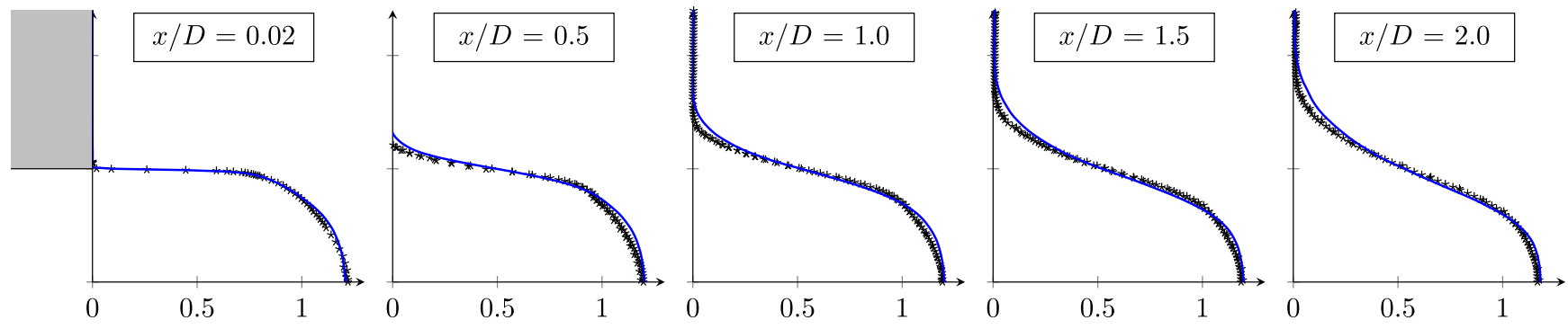

$\left\langle u_{x}\right\rangle / U_{j}$
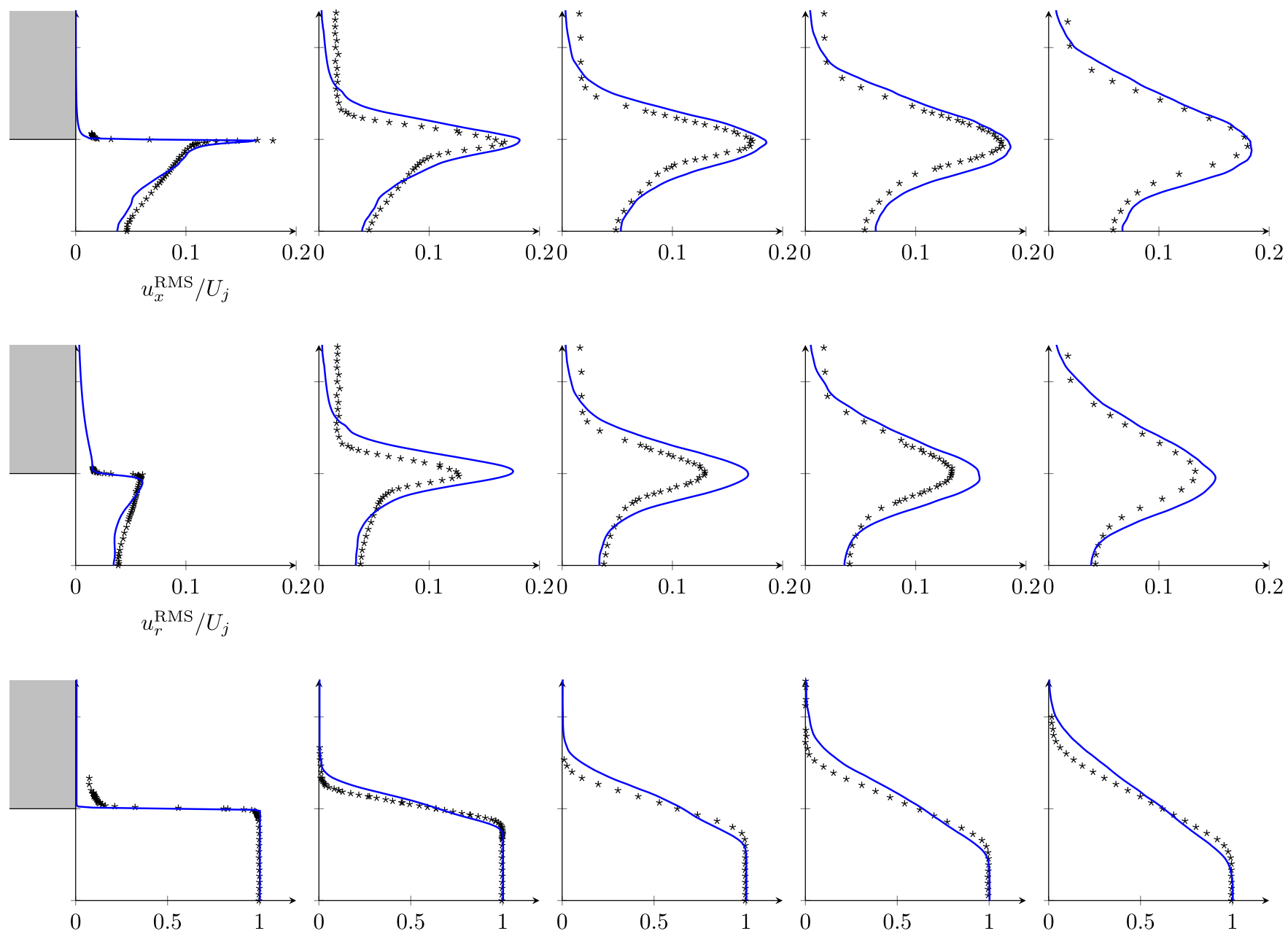

$\left(\langle T\rangle-T_{e}\right) /\left(T_{j}-T_{e}\right)$
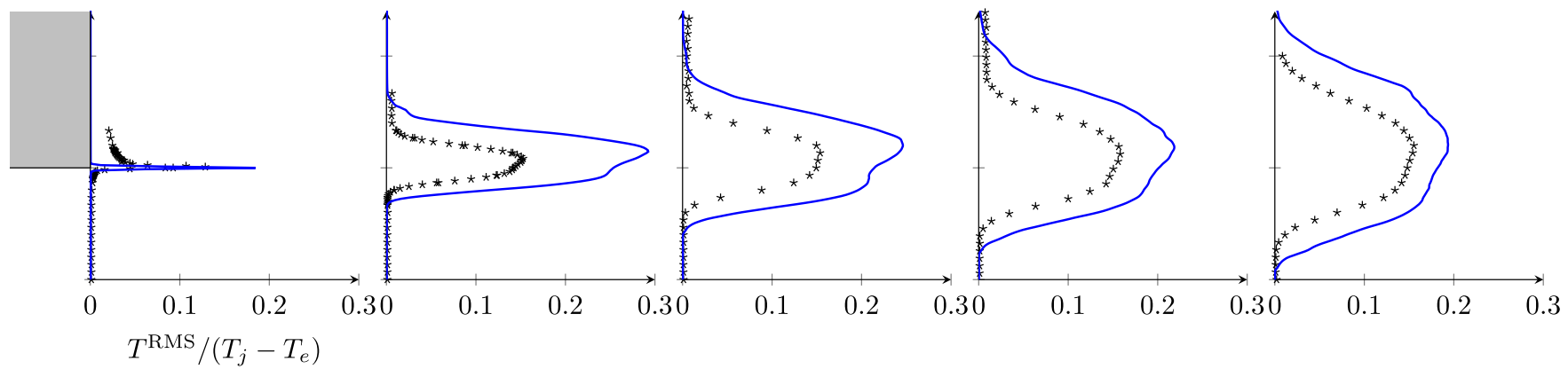

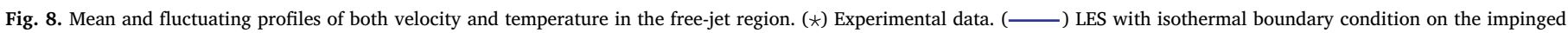
plate $\left(T_{w}=25^{\circ} \mathrm{C}\right)$. 


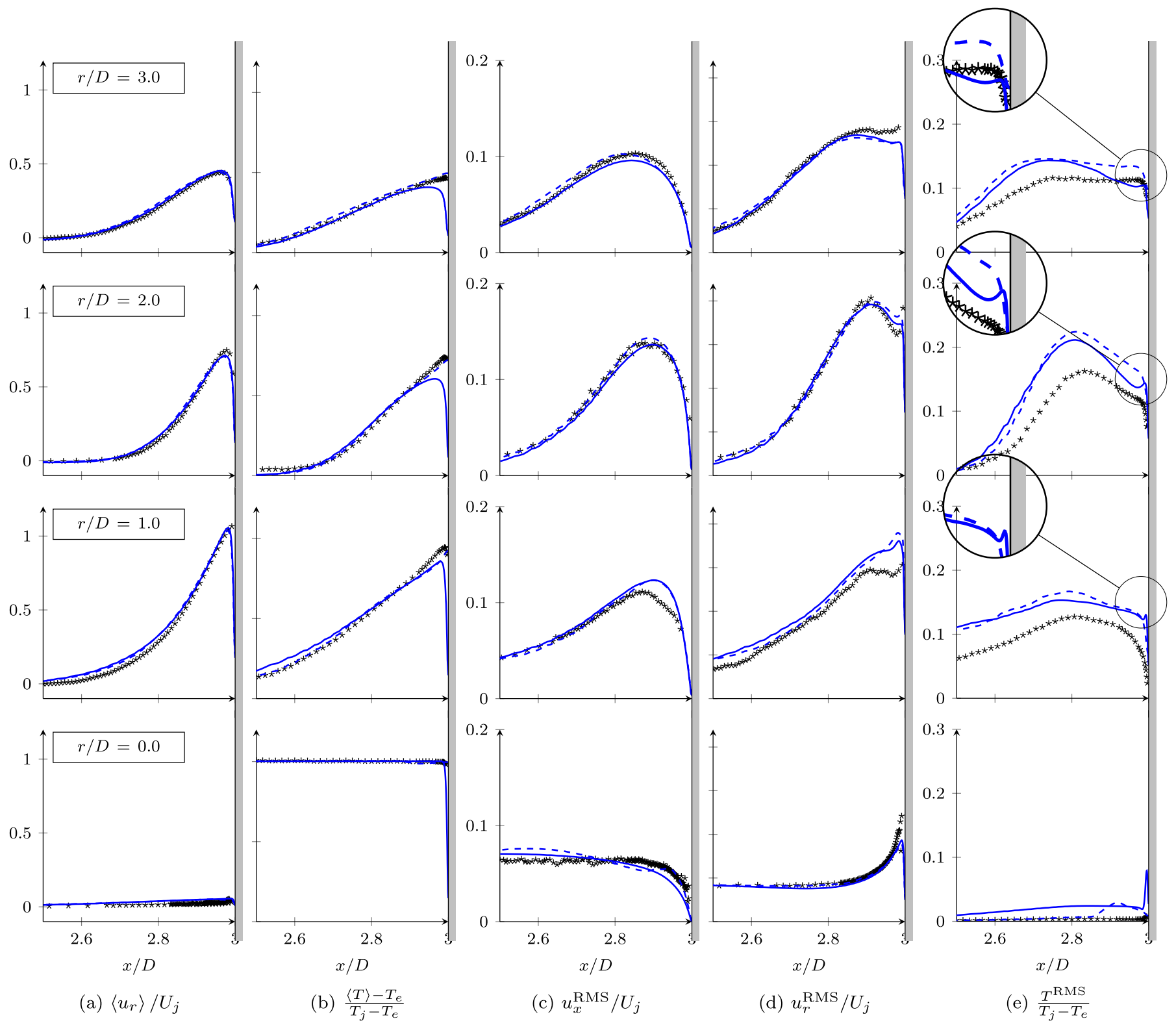

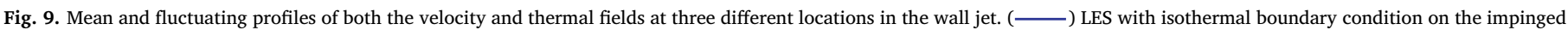

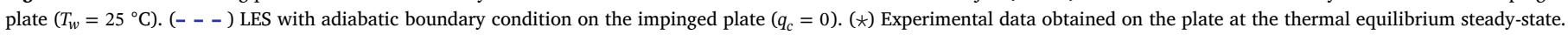

radial locations $r / D \geq 2$. No universal log-law ( $\kappa=0.41$ and $\operatorname{Pr}_{t}=0.9$ ) can be distinguished in the inner layer, contrary to the observations of Guerra et al. (2005) for a $\mathrm{Re}_{D}=35000$ impinging jet. This may be attributed to the fact that, in the present case, the inner layer is very thin. Indeed, the maximum radial velocity and temperature are reached at about 100 wall units, that is to say between the buffer layer and the beginning of the inertial sublayer. The outer layer is one order of magnitude thicker and extends up to $(H-x)^{+} \simeq 1000$.

\subsubsection{Wall transfer on the impinged plate}

The mean convective heat transfer coefficient $h$ is generally expressed by the Nusselt number $\mathrm{Nu}_{D}$, defined as:

$\mathrm{Nu}_{D}(r)=\frac{\langle h(r)\rangle D}{k_{f}}$

where $k_{f}$ is the thermal conductivity of the fluid. Fig. 11(a) shows that the Nusselt number in the stagnation region is well predicted whereas it is underestimated by $\sim 20 \%$ in the wall jet. We will come back later on the presumed origin of this discrepancy. The appearance of the secondary maximum at $r / D=2$ is nonetheless well reproduced by the simulation.

The adiabatic wall temperature can be rewritten in term of the effectiveness $\eta$, defined as:

$\eta(r)=\frac{T_{a w}(r)-T_{e}}{T_{j}-T_{e}}$

Fig. 11(b) shows that the computed effectiveness distribution compares satisfactorily with the experimental data. The effectiveness is close to one in the stagnation region, that indicates that the hot fluid which interacts with the plate at this location has not yet undergone mixing with ambient cold fluid. Further downstream the effectiveness decreases due to the mixing in the free-jet and the outer layer of the wall jet. This drop is slightly more pronounced in the LES because of the overestimation of the mixing in the free-jet shear layer.

The distribution of the mean wall friction modulus $\left\langle\tau_{w}(r)\right\rangle$ is plotted in Fig. 11(c). This quantity being not measured in our experimental data set, the data set of Alekseenko and Markovich (1996) $(H / D=3$ and $\operatorname{Re}_{D}=44000$ ) is used as validation purpose. Similarly to the Nusselt 


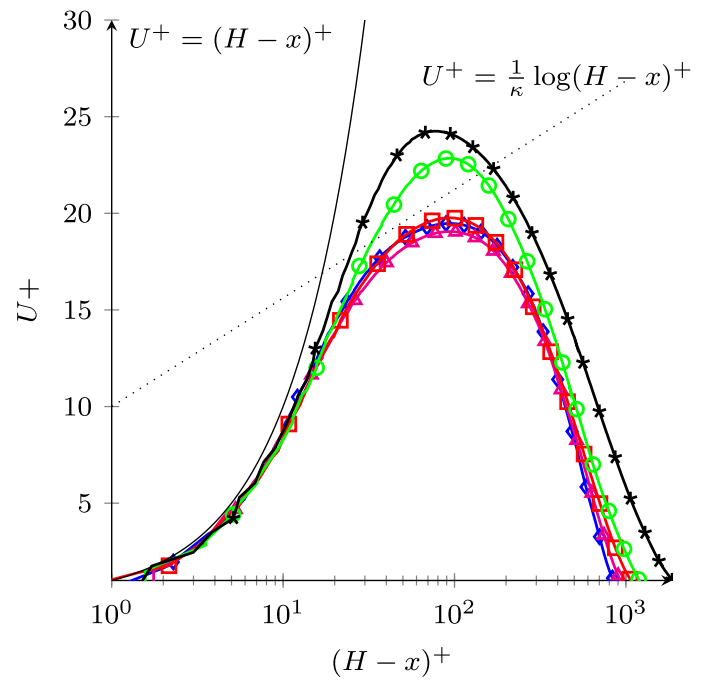

(a) Velocity.

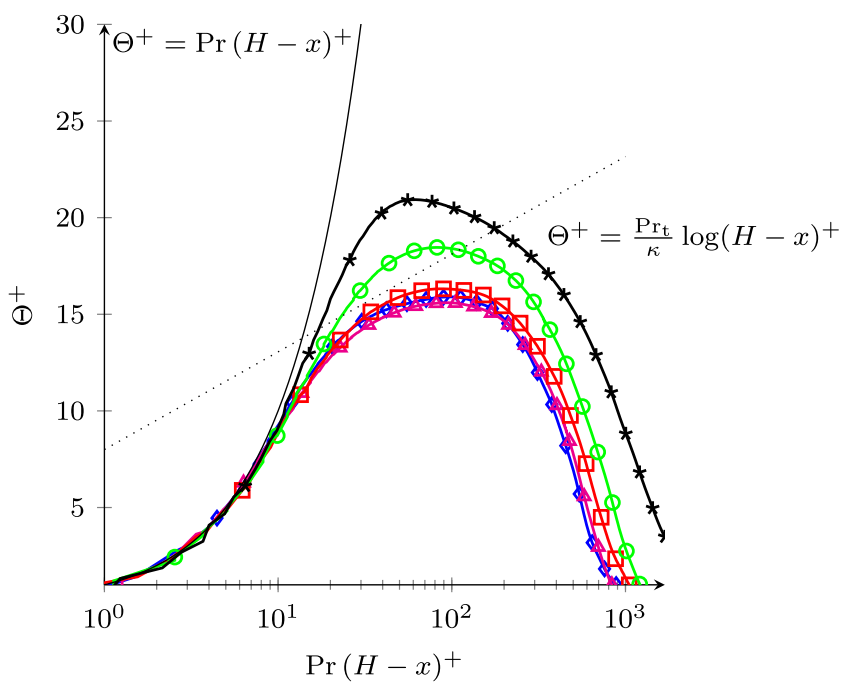

(b) Temperature.

Fig. 10. Velocity and temperature profiles at different radial locations scaled in wall unit. (Black stars) $r / D=1.0$. (Green circles) $r / D=1.5$. (Blue diamonds) $r / D=2.0$. (Magenta triangles) $r / D=2.5$. (Blue circles) $r / D=3.0$. Log-law constant has been chosen arbitrarily chosen.

number distribution, the mean friction modulus agrees well in the stagnation region. For higher radial locations, the predicted distribution features only a slight slope change (plateau) in the region $1.5<r / D<2$, while experimental data exhibit a secondary maximum in this region.

The comparison of Fig. 11(a) and (c) shows that the Reynolds analogy between the heat and mass transfer does not hold in the impinging flow: at the stagnation point the heat exchanges are the highest while the friction coefficient is equal to zero. Furthermore, the secondary peak of heat transfer does not match with the secondary peak (or the slope change for the LES) in the friction modulus distribution. The secondary maximum could then not be explained on the sole basis of this analogy.

\subsection{Spectral properties}

The spectral content of the numerical probes spread in the computational domain can be compared to the available experimental data that provide time-resolved measurements within the flow. The power spectral density (PSD) of velocity fluctuations at two representative

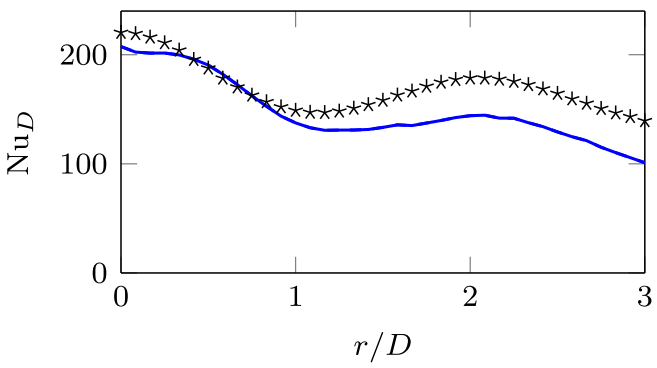

(a) Nusselt number $\mathrm{Nu}_{D}$.

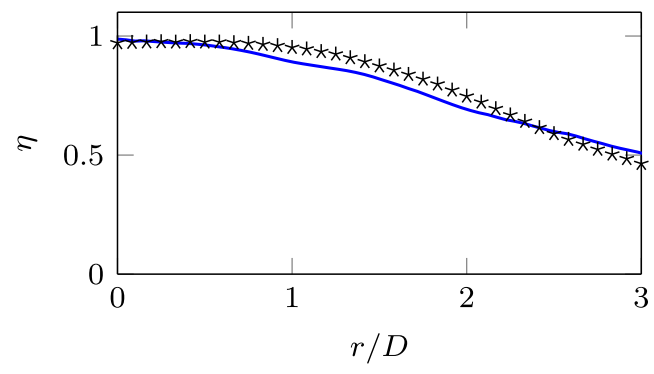

(b) Effectiveness $\eta$.

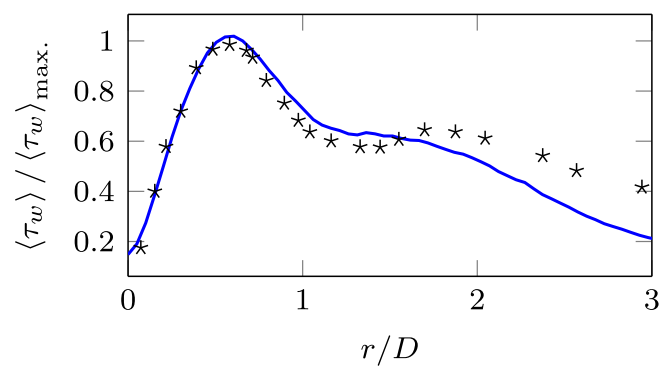

(c) Mean friction modulus $\left\langle\tau_{w}\right\rangle$ (experimental data from Alekseenko and Markovich [2]).

Fig. 11. Distribution of both the mean heat and momentum transfer on the impinged plate. (—) LES. ( $\star$ ) Experimental data.

locations in the jet is compared to the experimental data in Fig. 12. Due to the limited duration of the LES, the frequency resolution of the spectra, obtained through a Welsh method, is less than that available from the experiments $(\Delta f=3 \mathrm{~Hz})$. For the probe located in the jet shear layer (Fig. 12)(a), the agreement between spectra is satisfactory in the inertial frequency range, that is characterized by the $-5 / 3$ Kolmogorov law. The cut-off frequency of the simulation, which corresponds to the frequency at which PSD departs from the measurements, is of the order $f_{c}=2.5 \mathrm{kHz}$. This frequency can be related to the spatial wavelength $\kappa$ according to the Taylor's hypothesis which assumes a uniform convection speed $\langle u\rangle$ :

$\kappa_{c}=\frac{2 \pi f_{c}}{\langle u\rangle}$

It is shown in Fig. 12(a) that the cut-off wavelength multiplied by the local mesh size $\Delta$ corresponds approximately to $\pi / 3$. This demonstrates that the explicit LES filter dissipates the turbulent structures whose wavelength is not discretised by at least 6 cells, as expected.

In the jet core (Fig. 12)(b), the spectrum from the LES exhibits a frequency peak around $f_{p}=190 \mathrm{~Hz}$ (with spectral resolution of $17 \mathrm{~Hz}$ ), corresponding to a Strouhal number $\mathrm{St}_{D}=0.44$. This peak corresponds to column mode of the free-jet evidenced in the experiments but at a slightly lower frequency of $f_{p}=170 \mathrm{~Hz}\left(\mathrm{St}_{D}=0.4\right)$. 


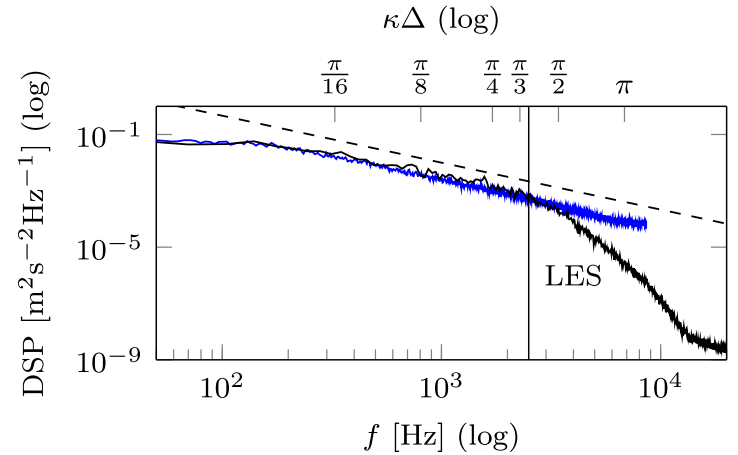

(a) PSD of $u_{r}$ for a probe located in the free-jet shear layer $(x / D=1.5, r / D=0.5)$.

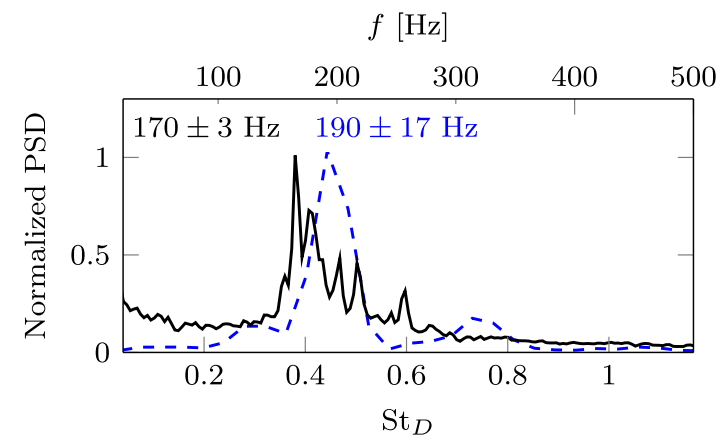

(b) PSD of $u_{x}$ for a probe located on the free-jet axis

$$
(x / D=1.5, r / D=0) \text {. }
$$

Fig. 12. Power spectral density (PSD) of the velocity at two locations in the flow field. (Dashed blue line) LES. (Solid black line) Experimental data. (For interpretation of the references to color in this figure legend, the reader is referred to the web version of this article.)

\section{Origin of the secondary maximum}

The previous section has shown that the present large-eddy simulation reproduces well the experimental case both for the statistical fields and the spectral content. This gives the confidence one expects to employ the 3D and unsteady data in order to scrutinize the mechanisms associated with the emergence of the secondary maximum in the Nusselt number distribution. This analysis will be principally based on the instantaneous distribution of the convective heat flux coefficient $h$ $(r, \theta, t)$. This quantity is computed from the instantaneous convective heat flux from the LES $q_{c}(r, \theta, t)$ and the mean adiabatic temperature $T_{a w}(r)$ (Fig. 11)(b) obtained from the LES-ADIA simulation, following:

$h(r, \theta, t)=\frac{q_{c}(r, \theta, t)}{T_{a w}(r)-T_{w}} \quad$ with $\quad T_{w}=25^{\circ} \mathrm{C}$

As outlined in the introduction, several authors (Popiel and Trass, 1991; Hadžiabdić and Hanjalić, 2008; Dairay et al., 2015) linked the secondary maximum to the unsteady separation phenomenon. For this reason, the appearance of "seperated" flow regions on the impinged plate will be also closely examined. These regions are defined as the surfaces where the radial component of the friction vector $\overrightarrow{\tau_{w}}$ is negative:

$\tau_{w, r}<0$

\section{1. $3 D$ flow organisation}

The instantaneous 3D flow organisation can be highlighted by means of isosurfaces of both static pressure and Q-criterion (Fig. 13).
The isosurfaces of static pressure permit to extract the large-scale structures in the flow. Two roll-up vortices (1) are evidenced in the neighbourhood of the pipe exit. They issue from the natural shear layer instability (Kelvin-Helmholtz) and exhibit a strong azimuthal coherence. As they are convected downstream, those vortices are likely to merge while they progressively lose coherence (2). When their remnants strike the plate, the flow tends to recover a strong azimuthal coherence with the formation of a large-scale primary structure (3). As the latter travels outwards along the plate, it loses again coherence, that is highlighted by the small pockets of pressure isosurface (4) clustered around the radial position $r / D=2.7$. These observations corroborate the findings of Hall and Ewing (2006) who observed from wall pressure measurements that azimuthal modes 0 and 1 were predominant in a high Reynolds $\left(\operatorname{Re}_{D}=50000\right)$ impinging jet $(H / D=2)$.

The isosurfaces of $Q$-criterion enable to isolate the smaller scales in the flow. Braid-like streamwise structures are evidenced between consecutive free-jet toroidal vortices (1) and (2)). It is shown in Fig. 13(a) that the primary structures are accompanied by numerous small-scale structures in the vicinity of the impinged plate. When the primary structure is located around the radial position $r / D=1$, these structures are mainly oriented in the azimuthal direction (loupe (i) in Fig. 13)(a). On the contrary, most of them are aligned with the mean flow direction when the primary structure is located further downstream (loupe (ii) in Fig. 13)(a), featuring a streak-like behaviour.

\subsection{Instantaneous wall transfers}

The instantaneous distribution of the "separated" flow regions on the impinged plate is represented in Fig. 13(a). First of all, this indicates that flow separation occurs at this high Reynolds. Next, it seems that those regions are located beneath the primary structures but do not exhibit the azimuthal coherence that have been highlighted in the DNS of Dairay et al. (2015) $\left(\operatorname{Re}_{D}=10000\right)$ and the LES of Aillaud et al. (2016) $\left(\operatorname{Re}_{D}=20000\right)$. In the present case, the "separated" regions look rather like small isolated pockets, that are convected with the primary structure.

The instantaneous distribution of the heat flux coefficient is shown in Fig. 13(b). At this particular instant, the primary structure is located near the radial position $r / D=2$. This visualisation shows that the convective heat flux coefficient exhibits locally very high values (up to three times higher than the mean value of $h$ at the stagnation point), underneath the primary structure. These small-scale regions correspond to the hot spots observed in the previous works of Uddin et al. (2013) and Dairay et al. (2015).

\subsection{Primary structure footprint}

The previous section has shown that flow separations and hot spots appears in the neighbourhood of the primary structures. This section aims to quantify the footprint of a primary structure, in terms of both friction and heat, when it travels along the plate. For that purpose, azimuthal averaging of the instantaneous distributions is performed. This approach is justified by the fact that the primary structure exhibits a strong azimuthal coherence. Three new quantities are then defined:

- the azimuthal mean of the heat transfer coefficient:

$$
\langle h\rangle_{a z .}(r, t)=\frac{1}{2 \pi} \int_{0}^{2 \pi} h(r, \theta, t) \mathrm{d} \theta
$$

- the azimuthal mean of the friction vector radial component:

$$
\left\langle\tau_{w, r}\right\rangle_{a z .}(r, t)=\frac{1}{2 \pi} \int_{0}^{2 \pi} \tau_{w, r}(r, \theta, t) \mathrm{d} \theta
$$

- the total friction "force" due to the separated regions: 


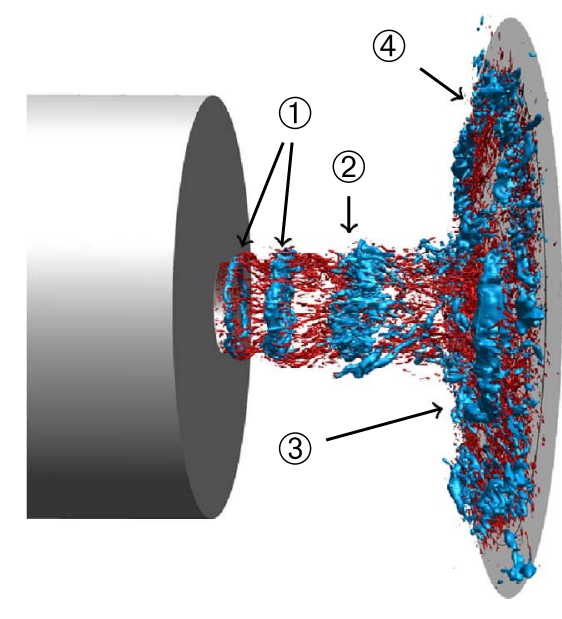

(a) Side view at instant $t=8.8 \tau_{c}$.

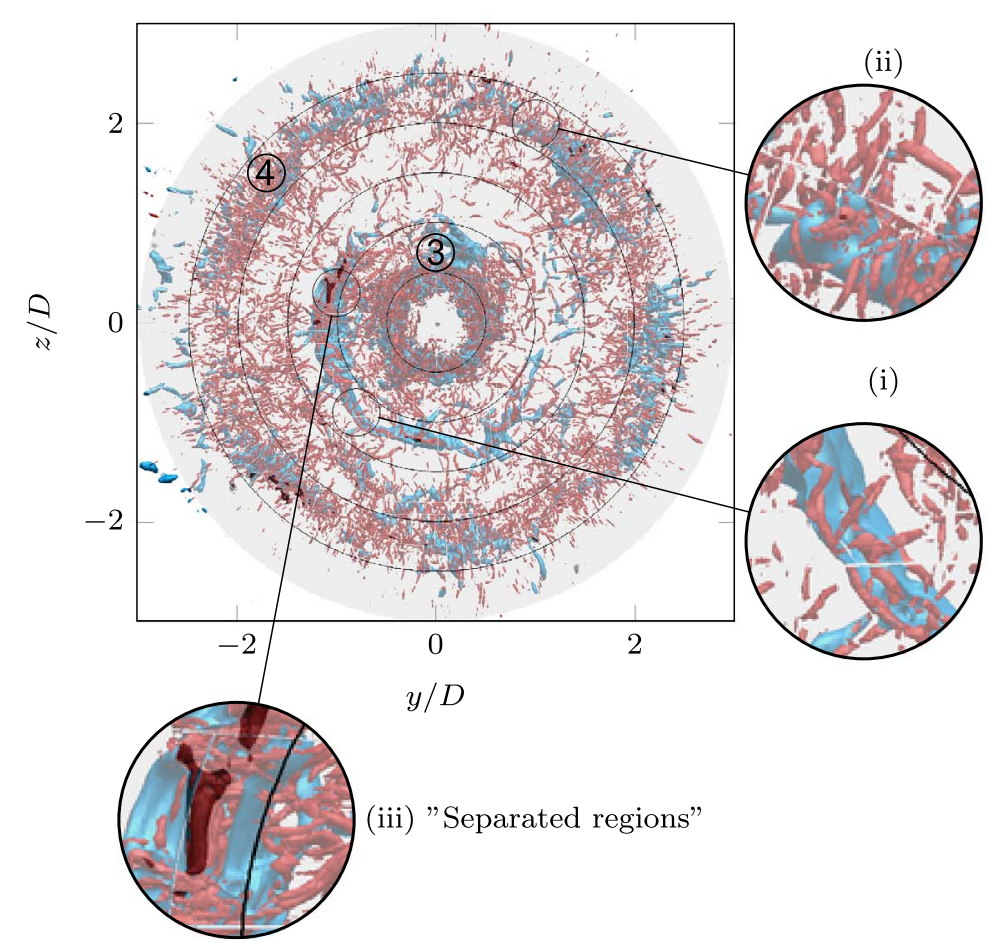

(c) View from the rear face of the plate at instant $t=8.8 \tau_{c}$. (Black area) "Separated" regions. (Black circles) Iso- $r$ position $(r / D=0.5,1,1.5,2,2.5$ and 3)

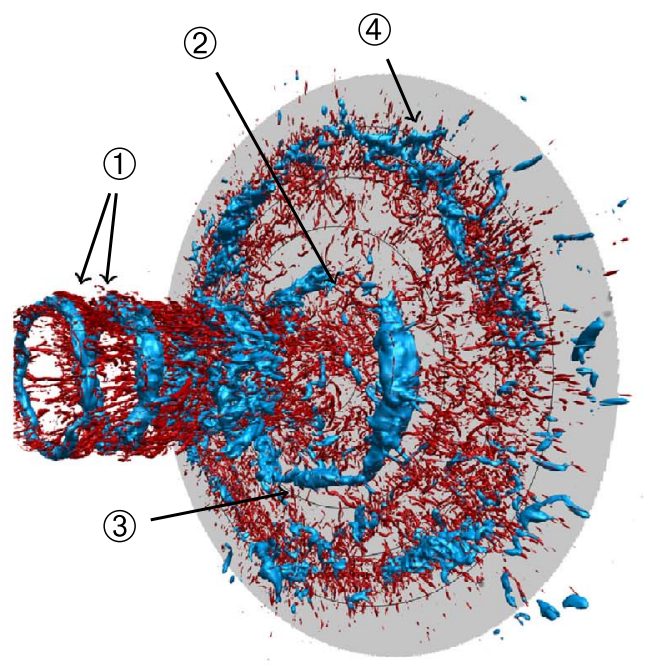

(b) Front view at instant $t=8.8 \tau_{c}$.

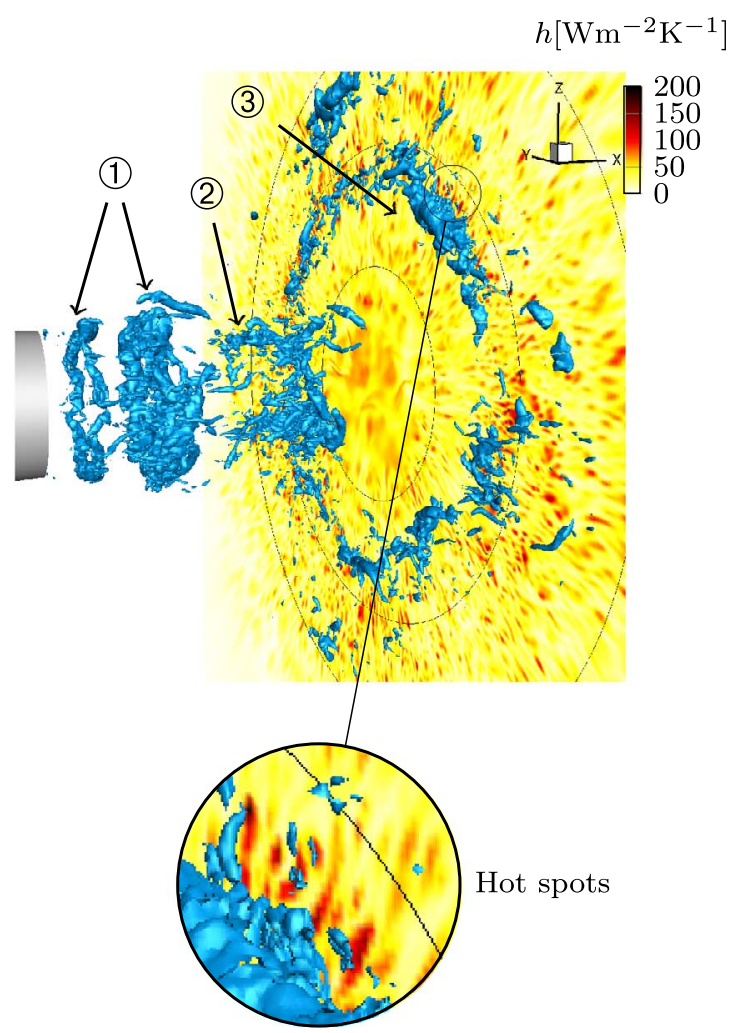

(d) Convective heat transfer coefficient distribution at instant $t=10.6 \tau_{c}$. (Black circles) Iso- $r$ position $(r / D=1,2$ and 3)

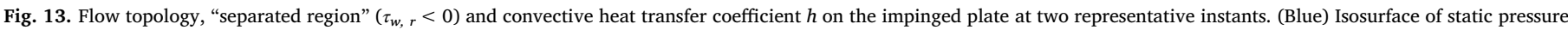
$p_{e}-p=40 P a$. (Red) Isosurface of $Q$-criterion.

$$
F_{\text {sep }}(r, t)=\int_{0}^{2 \pi} \min \left[\tau_{w, r}(r, \theta, t), 0\right] r \mathrm{~d} \theta
$$

The spatio-temporal maps of those quantities are represented in Fig. 14. In the stagnation region (between $r / D=0$ and $r / D=1$ ) the convective heat transfer coefficient varies little and remains close to its mean value $\langle h\rangle$ (Fig. 14)(a). At the opposite, one can clearly distinguish a regular increase in the convective heat transfer coefficient in the wall- jet region (between $r / D=1$ and $r / D=3$ ). This increase is the highest at the radial location $r / D=2$, which corresponds to the location of the secondary maximum. In Fig. 14(a), the increase reproduces 13 times, with a period during two consecutive events approximately equal to the characteristic time $\tau_{c}$ associated to the free-jet column mode $\mathrm{St}_{D}=0.44$. From the experimental data, we pointed out that the shedding of the primary structures is characterized by the same frequency as the free-jet column mode. This demonstrates that the secondary maximum, which occurs periodically, is strongly related to the passage of a primary 

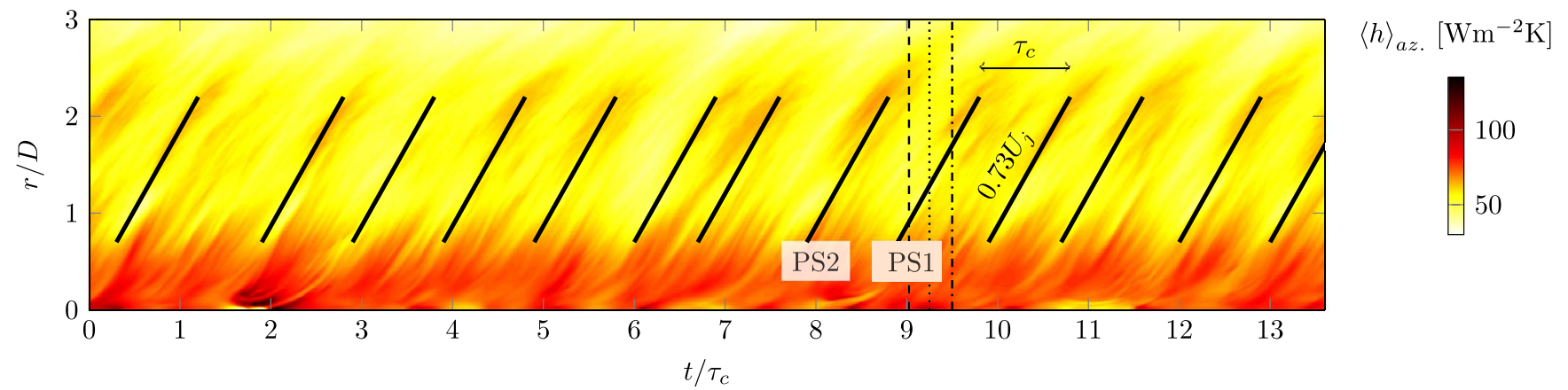

(a) Azimuthally-averaged convective heat transfer coefficient.

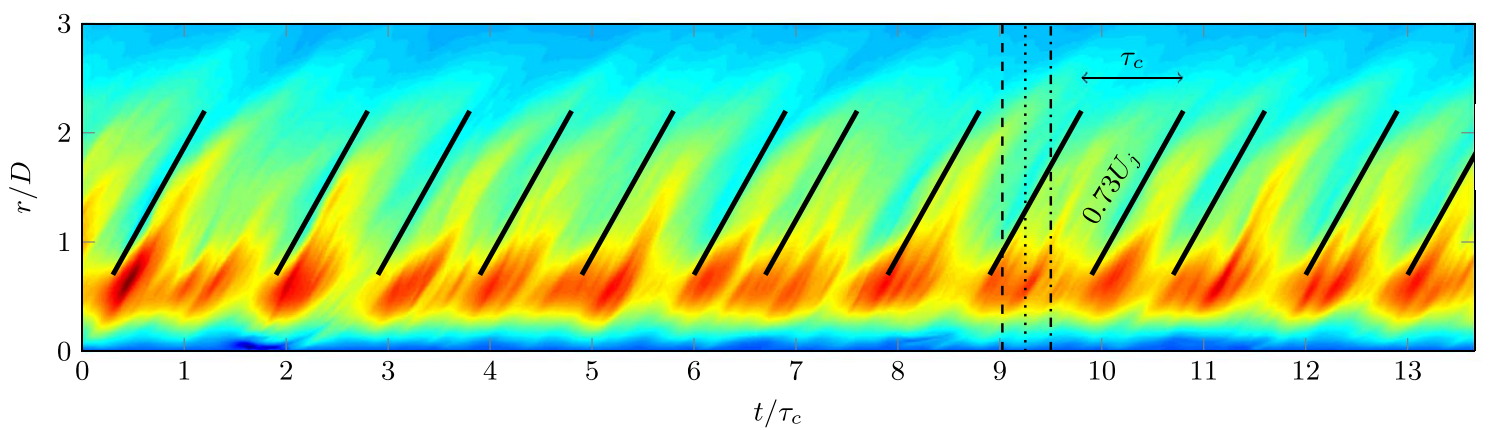

$\left\langle\tau_{w, r}\right\rangle_{a z .}\left[\mathrm{Nm}^{-2}\right]$

(b) Azimuthally-averaged wall friction.
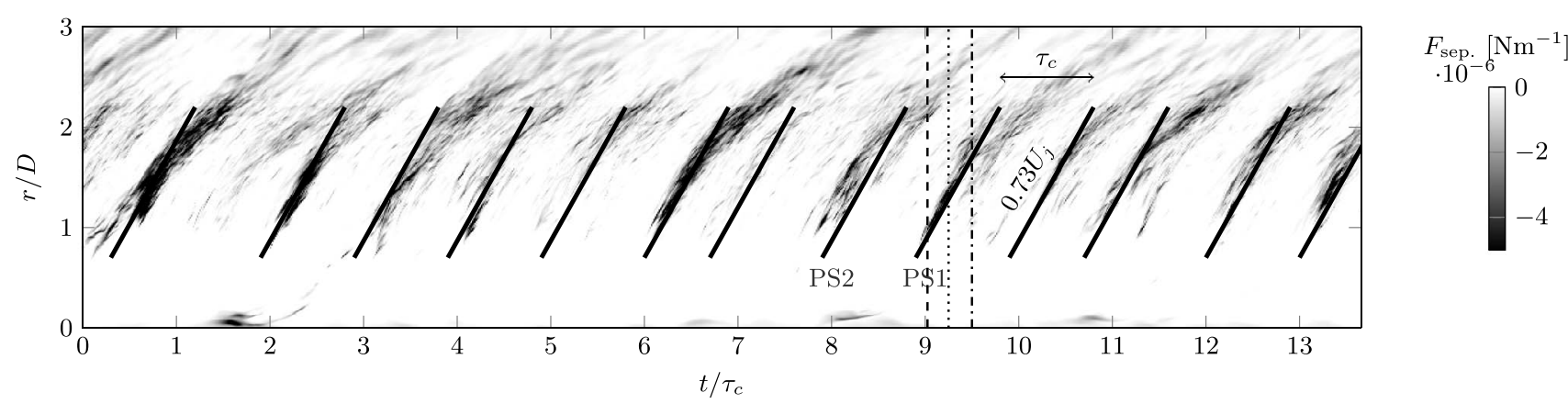

(c) Total "friction" force.

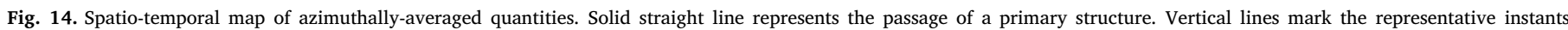
corresponding to the instantaneous distributions depicted in Fig. 15.

structure above the impinged plate. The straight lines in Fig. 14 mark three representative passages of a primary structure, the slope corresponding to the convection velocity.

Fig. 14(b) shows that the passage of a primary structure can be linked with a decrease in the azimuthally-averaged friction coefficient in the region between $r / D=1$ and $r / D=2$.

The total friction force $F_{\text {sep }}$ due to the separated regions allows to quantify the intensity of separation at a given radial location. Fig. 14(c) indicates that separation appears regularly from the radial location $r / D=1$ at the shedding frequency of the primary structures. The separation intensity is the highest between $r / D=1$ and $r / D=2$.

By comparing the three azimuthally-averaged quantities along the straight lines in Fig. 14, one can see that the presence of separated regions cannot be unequivocally linked to an increase or decrease in the heat transfer coefficient. When the primary structure is located at $r / D=1$, the separation corresponds to low heat transfer whereas it corresponds to high heat transfer when the primary structure is located at $r / D=2$. Conversely, the decrease in the friction coefficient can be related to the presence of separated regions. Unsteady separation could then explain the dip between the two maxima in the wall friction distribution (Fig. 11)(c).

In order to highlight the possible link between heat increase and separation, the instantaneous distribution of the convective heat transfer coefficient and the separated regions are represented in Fig. 15 for three different instants. The isosurfaces of static pressure above the plate, represented by translucency, allow to locate the position of the primary structures. These three instants have been selected to follow the convection of two primary structures:

- Structure PS1, which travels from its impact at $r / D=1$ to the radial location $r / D=2$;

- Structure PS2, which travels from the radial location $r / D=2$ to $r / D=2.5$.

At the instant $t / \tau_{c}=9.02$ (Fig. 15)(a), the structure PS1 is located at $r / D=1$. As already highlighted in Fig. 13(a), the interaction of this structure with the wall generates unsteady separation pockets. Those separations do not cause any heat increase or decrease at this radial location, as evidenced by the loupe in Fig. 15(a). As the structure PS1 progresses outwards, one can observe emergence of many hot spots 

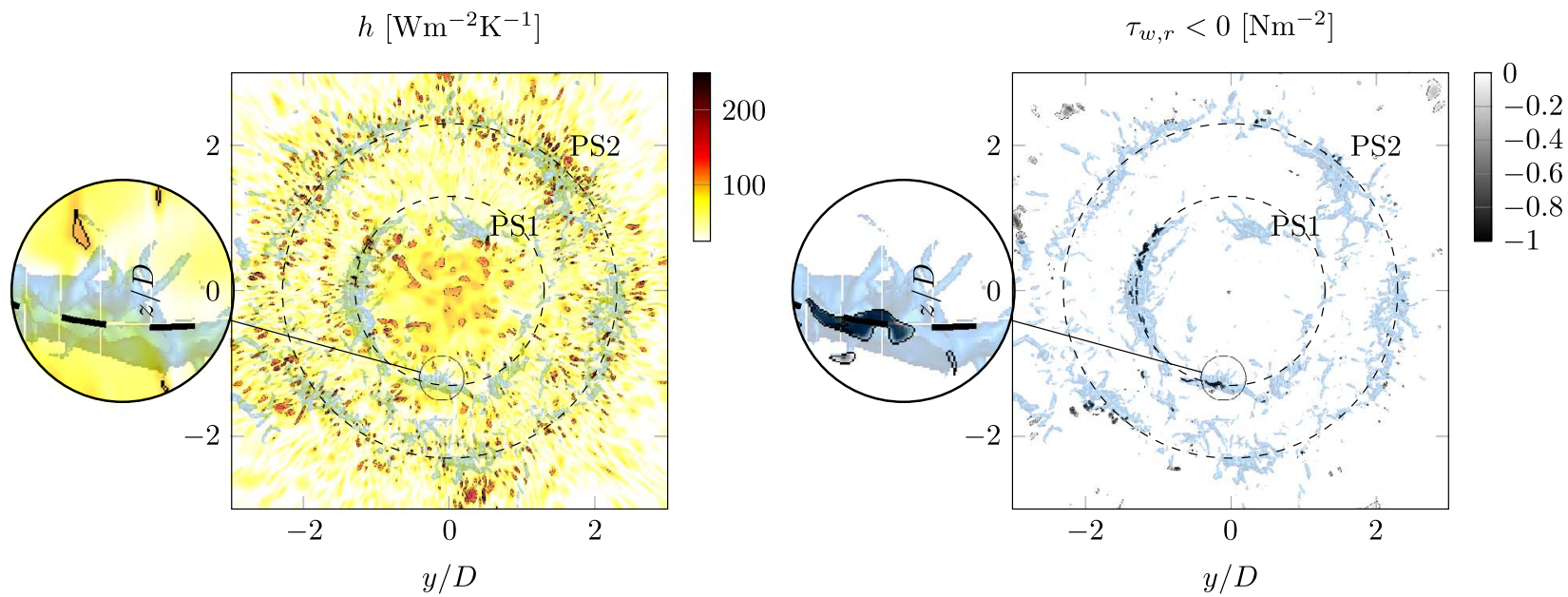

(a) $t / \tau_{c}=9.02$
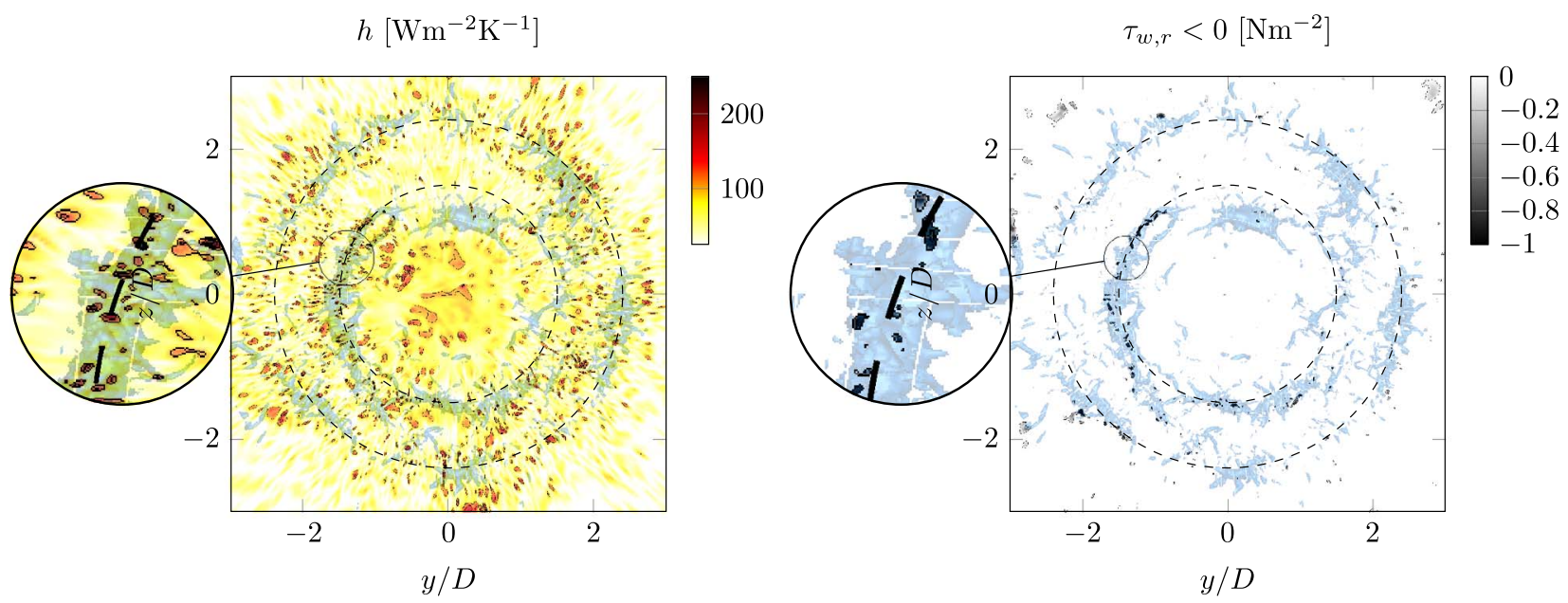

(b) $t / \tau_{c}=9.25$
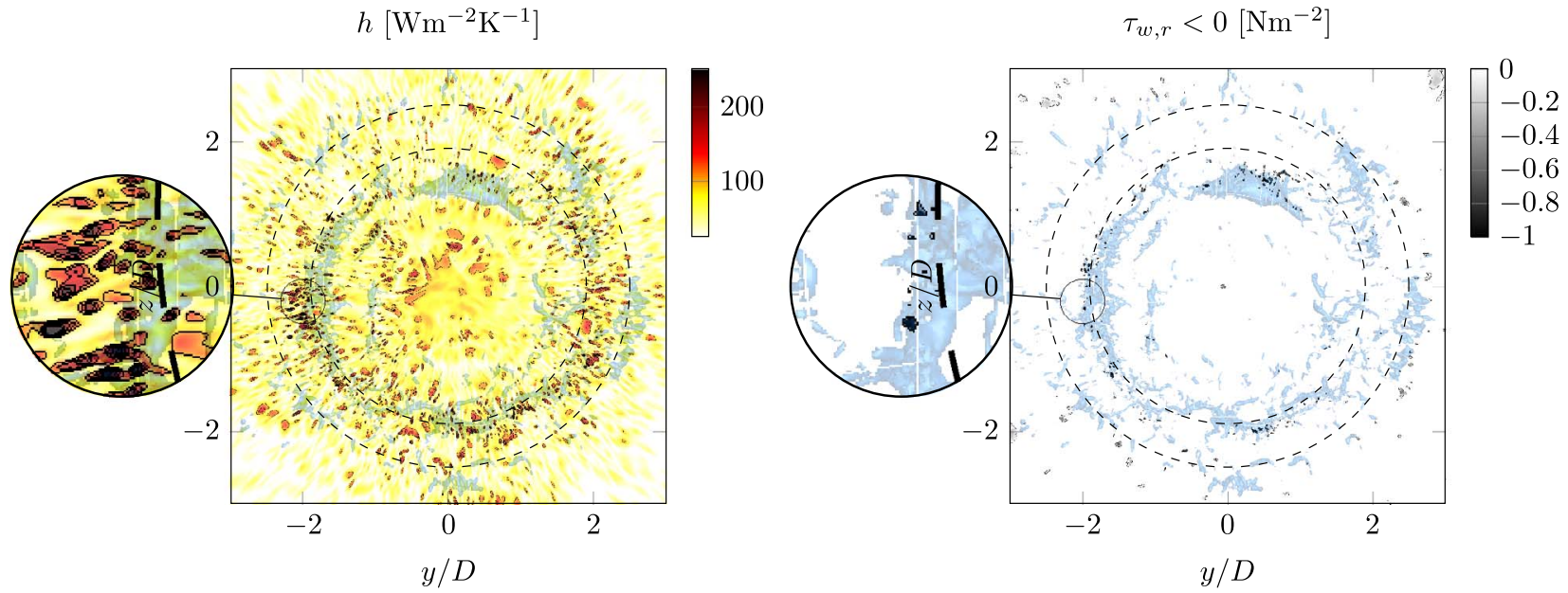

(c) $t / \tau_{c}=9.5$

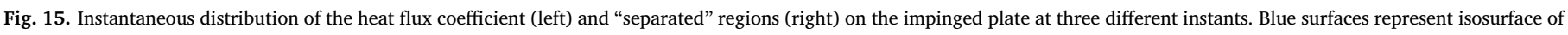
static pressure in the field above the plate. Dashed circles mark the radial position of the two primary structures.

underneath (Fig. 15(a), left). The number and the intensity of these hot spots are the highest when the primary structure is located around $r / D=2$ (Fig. 15(b), left), the position at which the azimuthally-averaged heat flux coefficient increases. For higher radial location, the hot spots disappear and decrease in intensity as evidenced by the structure PS2. Fig. 15(a)-(c) (left) show that the separated pockets break out when they travel with the primary structure. If some hot spots can be related to separated regions (loupes in Fig. 15(b) and (c)), the majority 
of them cannot be explained from this simple analysis and further investigation of the local flow dynamics is required.

\subsection{Probability density function}

Prior to take a closer look at the local flow dynamics in the vicinity of the hot spots, their role in the emergence of the secondary maximum is quantified by means of a probability density analysis. Following the approach adopted by Dairay et al. (2015), the probability density function (PDF) of the convective heat transfer coefficient $h$ is computed for each radial location along the wall. Formally its reads as:

$\operatorname{PDF}(h, r)=\mathscr{P}(h(t, \theta)=h)$ for given $r$

The $\operatorname{PDF}(h, r)$ map is represented in Fig. 16, where the mean heat flux coefficient distribution $\langle h\rangle$ and its most probable value $h\left(\mathrm{PDF}_{\text {max. }}\right)$ are also plotted. In the stagnation region (between $r / D=0$ and $r / D=1$ ), it can be seen that the PDF is symmetrical with respect to the mean value of $h$, as detailed in Fig. 16 (left). From the radial location $r / D=1$, the PDF loses symmetry with appearance of strong events of high heat transfer coefficient. The asymmetry is the strongest at $r / D=2$, which corresponds to the position of the secondary maximum in the mean heat transfer coefficient. At this location the mean heat transfer coefficient is higher than its most probable value (Fig. 16). Further downstream, the PDF retrieves a certain symmetry. This analysis clearly demonstrates that the mean distribution of $h$ is pulled up by the intense thermal events produced by the hot spots, that finally induce the secondary maximum. This corroborates the findings of Dairay et al. (2015) obtained for a low-Reynolds number configuration.

In the present simulation, we have observed that the smallest hot spots are discretised on few cells only. This suggests that smaller hot spots might not be correctly resolved or not resolved at all. That could explain why the mean Nusselt number distribution is underestimated with respect to the measurements for radial location higher than $r / D=1$ (Fig. 11)(a). The wall-resolved LES criterion of Piomelli and Chasnov (1996) may then not be suitable to capture the mechanisms leading to the secondary maximum, as already suggested by Hadžiabdić and Hanjalić (2008).

\subsection{Focus on the hot spots}

Since the relation between hot spots and the secondary maximum has been demonstrated, the next step is to isolate the flow mechanisms that lead to this intense increase in the heat transfer coefficient.

\subsubsection{Friction lines}

In order to relate the hot spots to the local velocity field, the friction lines in the vicinity of representative hot spots are analyzed. One reminds that are defined as the streamlines of the vector field $\tau_{w}$ and can be interpreted as the projection of the near wall velocity vector. From the observation of a large amount of hot spots, two types can be distinguish. Fig. 17 shows the friction line patterns observed in the vicinity of the two types of hot spots. The mean flow direction, which is the same as the radial direction, at the location of the hot spot is represented by an arrow.

The hot spots of type I, an example of which is depicted in Fig. 17(a), are akin to a local jet impingement, with friction lines spreading outward from the hot spot center. In the vicinity of the spot some friction lines are then oriented upstream of the mean flow direction, which indicates a flow reversal. Based on the definition (16) of the separation, this type of hot spot corresponds to a "separated" flow region. It can also be seen that the "separation" and reattachment lines are orthogonal to the radial direction. This type is very similar to those evidenced by Dairay et al. (2015) by means of conditional averaging of the aerodynamic fields.

The second type of hot spot is the most encountered. Two examples of them are represented in Fig. 17(c) and (b). They reveal that the friction lines strongly spread from each other in the vicinity of the heat transfer enhancement. In certain cases, the spreading is so intense that an impingement line oriented with the mean flow direction is visible (Fig. 17)(c).

It turns out that the common feature shared by the two kinds of spot is that the friction lines exhibit an intense divergence above them. The friction line divergence can be related to the downwash motion due to velocity vector oriented to the wall. The positive axial velocity causes the thinning of the thermal boundary layer and the enhancement of the heat transfer coefficient. These observations are consistent with the findings of Aillaud et al. (2016) who showed that the convection of cold fluid onto the hot plate was the most probable event in the region of the secondary maximum.

\subsubsection{Local aerothermal field}

The interest is now attracted to the origin of the downwash motion around the hot spots. For that purpose, one will take a closer look to the flow velocity field just above a representative hot spot. This is done thanks to a cylindrical slice in the flow field at a given radial location $r / D=2$ (Fig. 18)(a). Pseudo-streamlines of the velocity field are drawn on the cylindrical slice along with the instantaneous static temperature field. A close-up view at the intersection between the cylindrical slice and the impinged plate is presented in Fig. 18(b). The friction lines on the impinged plate are also represented. The loupe in Fig. 18(b) illustrates that the pseudo-streamlines in the slice which are oriented towards the wall induce the divergence of the friction line on the plate. It can also be seen the effect of the downwash on the thermal boundary layer thickness. The pseudo-streamlines in Fig. 18(b) indicate that the downwash motion is produced by elongated counter-rotating structures whose vorticity vector is oriented in the streamwise direction. They can be related to the streaks-like structures that have been shown to accompany the primary structure in Fig. 13(c).

A new question arises from these findings : why are the streaks-like structures more numerous and/or intense in the region at which the secondary maximum emerges? To answer this question, one must come back on the dynamics of the large-scale primary structures.

\subsection{Primary structure trajectory}

In the experiments, a vortex-region detection algorithm were applied to the instantaneous velocity fields measured by stereo-PIV in order to highlight the mean path of the primary structures. This procedure has been applied to the $O x y$ and $O x z$ slices that have been extracted from the LES. The location of the center of the detected vortical

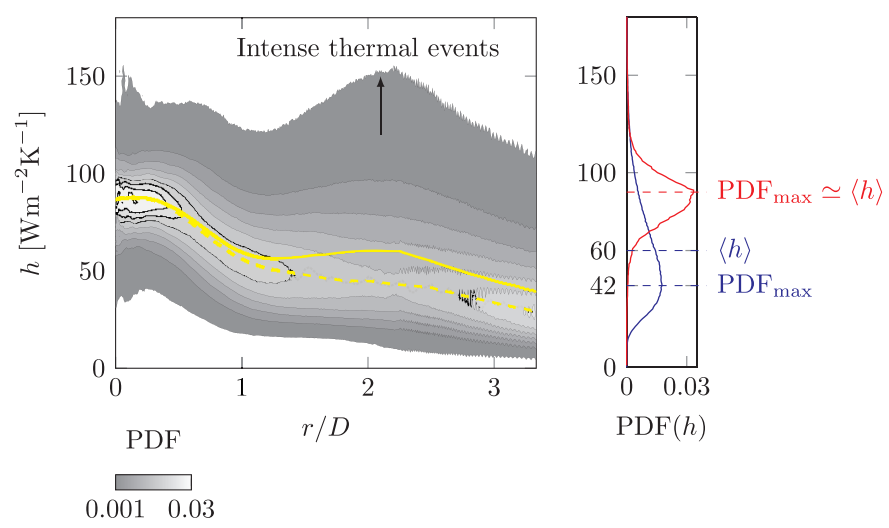

Fig. 16. Probability density function (PDF) of the convective heat flux coefficient $h$ at different radial positions $r / D$ along the impinged plate. (Right) Map the probability density function. (Solid line) Time averaged value of $h:\langle h\rangle$. (Dashed line) Most probable value of $h: h\left(\mathrm{PDF}_{\text {max. }}\right)$. (Left) PDF at two radial locations. (Red lines) $r / D=0$, impingement point. (Blue lines) $r / D=2$, location of the secondary maximum. 


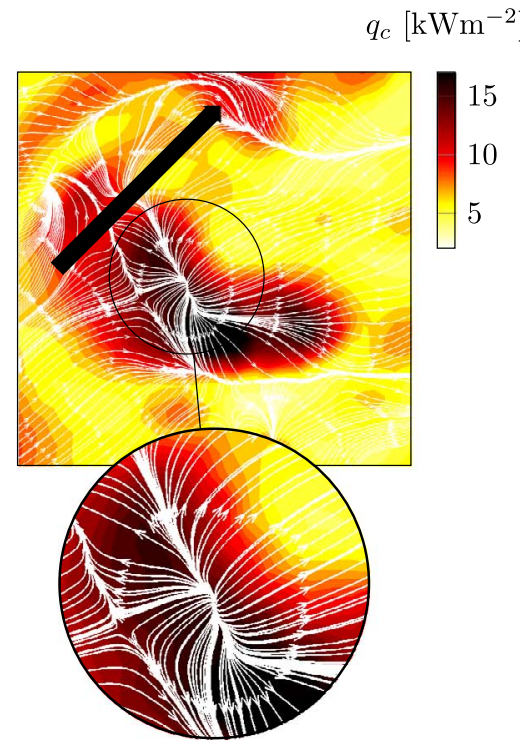

(a) Type I.

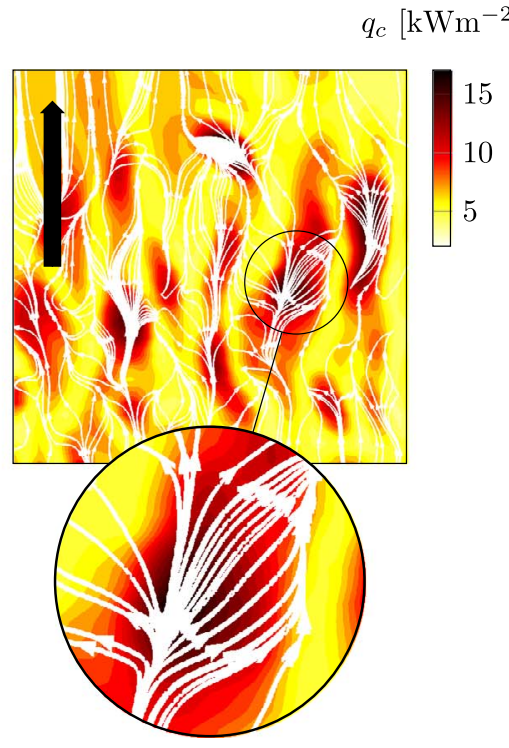

(b) Type II.
$q_{c}\left[\mathrm{kWm}^{-2}\right]$

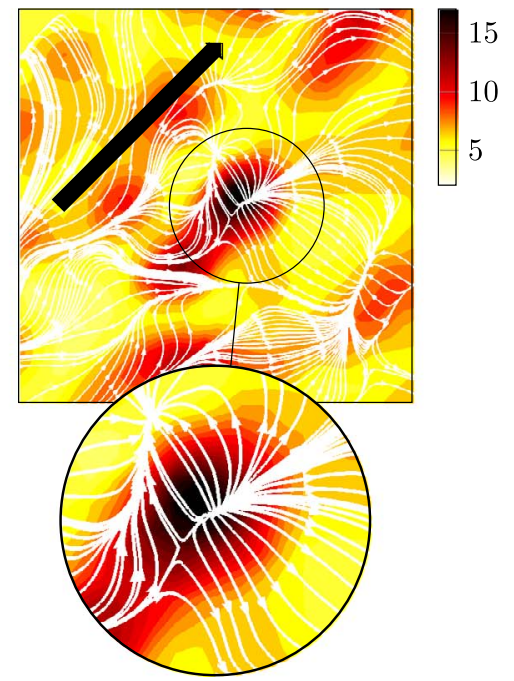

(c) Type II.

Fig. 17. Friction lines $\overrightarrow{\tau_{w}}$ around different kinds of representative hot spots located around the radial location $r / D=2$. The solid arrows represent the mean direction of the flow.

structures along with their sizes are gathered in Fig. 19. The mean path of the primary structure is also plotted. It turns out that the trajectory is in complete agreement with that obtained from the measurements. In particular, the rebound of the primary structure, which is the closest to the wall at the radial location $r / D=1.7$, is remarkably well reproduced.

The location of the rebound, sightly upstream of the location of the secondary maximum, suggests that the primary structure approach to the wall could be linked to an increase in the number and/or intensity of the streaks-like structures. The detailed mechanism is however still unclear. We may hypothesize some instability due to the adverse pressure gradient downstream of the accelerated flow region generated by the primary structure when it comes close to the wall.

\section{Conclusions}

A wall-resolved large-eddy simulation of a heated impinging jet has been performed for the first time at high Reynolds number. The simulation has been properly validated on a detailed experimental data base. The comprehensive data provided by this unsteady and wall-resolved simulation enabled to investigate which mechanism is responsible for the secondary maximum in the mean Nusselt number distribution. Based on instantaneous flow topology, azimuthally-averaged quantities and probability density function, the role played by hot spots of high convective heat transfer coefficient has been demonstrated. Only the hot spots of type I could be associated to unsteady separation, the others being related to an intense friction line divergence due to the downwash produced by streaks-like structures. Furthermore, the hot

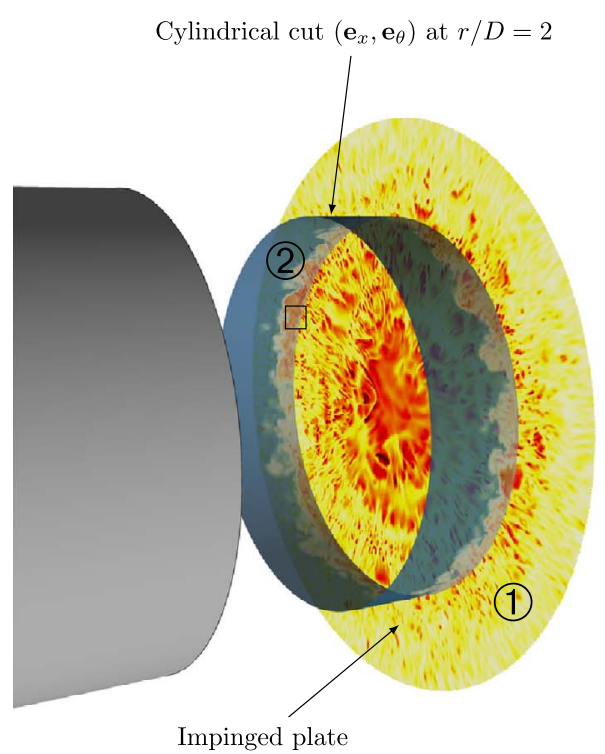

(a) Location of the selected surfaces.

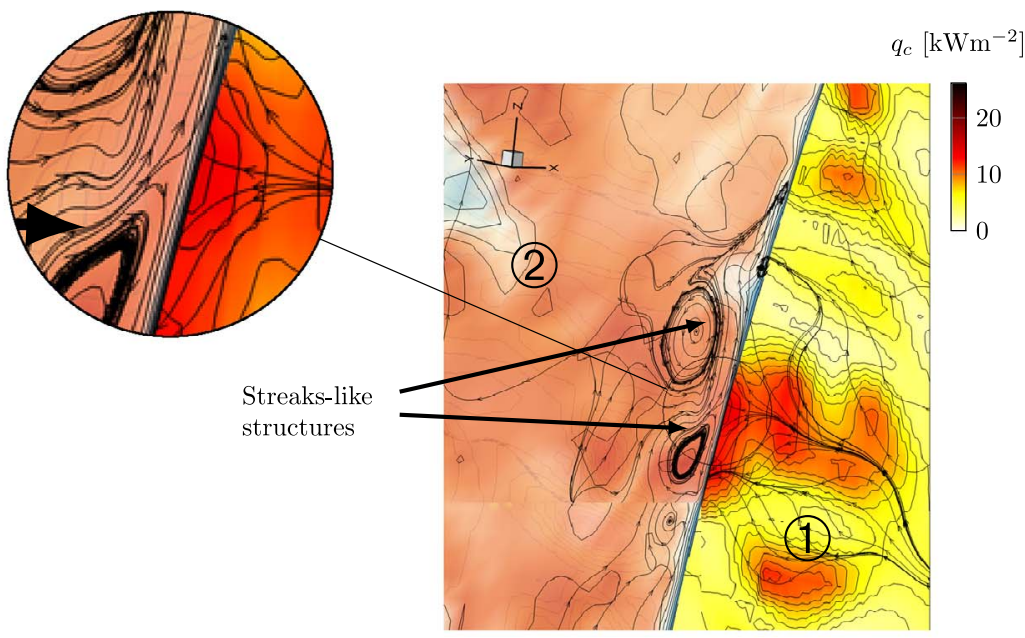

$$
\begin{aligned}
& \left(T-T_{e}\right) /\left(T_{j}-T_{e}\right) \\
& \begin{array}{llllllll}
0 & 0.2 & 0.4 & 0.6 & 0.8
\end{array}
\end{aligned}
$$

(b) Close-up view in the vicinity of the hot spot.

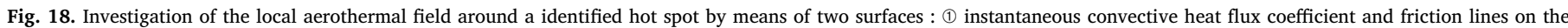
impinged plate (circular plane) and (2) instantaneous static temperature field $T$ and projected velocity streamlines in a cylindrical cut at the radial position $r / D=2$. 


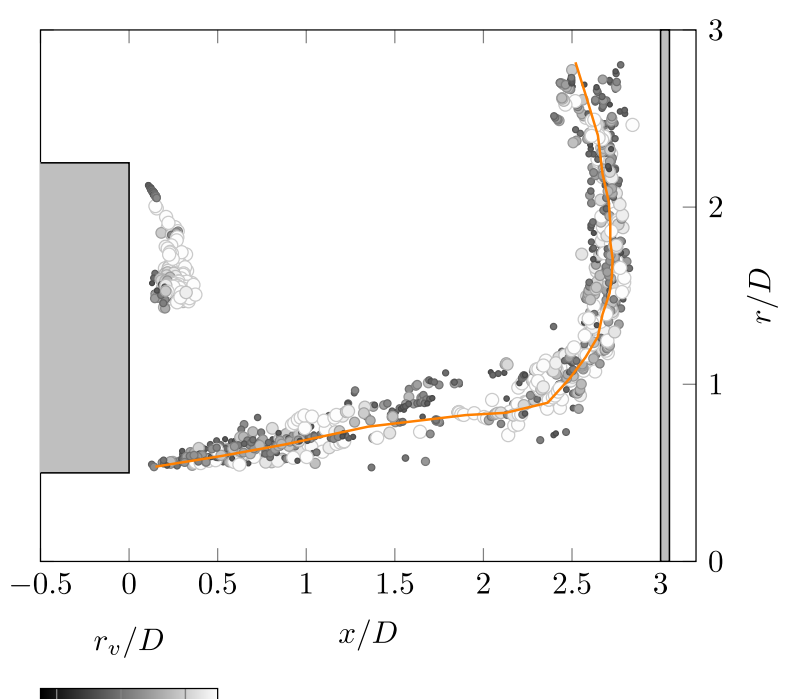

$0.1 \quad 0.2 \quad 0.3$

Fig. 19. Application of the vortex-region detection algorithm to instantaneous Oxy and Oxz slices from LES. Orange solid line represents the mean path of the detected vortical structures. Gray scale indicates the equivalent radius of the structures.

spot emergence have been linked to the passage of primary structure which sheds at the free-jet column mode and rebounds on the plate near the location of the secondary maximum. The picture is however still incomplete and further investigation should be undertaken to isolate the mechanisms that are responsible for the creation and/or intensification of the streaks-like structures near the radial location of the secondary maximum.

\section{Acknowledgments}

The present research was supported by Onera in the framework of the federative project "PRF JET". This work was performed using HPC resources from GENCI-CINES (Grant 20152a7373 and 20162a7373). The authors would particularly like to thank Philippe Reulet for his assistance in the experimental data base generation and Francois Chedevergne and Bertrand Aupoix for their insightful advice.

\section{References}

Aillaud, P., Duchaine, F., Gicquel, L., Didorally, S., 2016. Secondary peak in the nusselt number distribution of impinging jet flows: a phenomenological analysis. Phys. Fluids 28 (9), 095110.

Alekseenko, S.V., Markovich, D.M., 1996. Local characteristics of impinging round jets. Eng. Turb. Model. Exp. 3, 633-642.
Baughn, J.W., Shimizu, S., 1989. Heat transfer measurements from a surface with uniform heat flux and an impinging jet. J. Heat Transfer 111 (4), 1096-1098.

Bechara, W., Bailly, C., Lafon, P., Candel, S., 1994. Stochastic approach to noise modeling for free turbulent flows. AIAA J. 32 (3), 455-463.

Bogey, C., Bailly, C., 2006. Large eddy simulations of round free jets using explicit filtering with/without dynamic Smagorinsky model. Int. J. Heat Fluid Fl. 27 (4), 603-610.

Bogey, C., Bailly, C., Juvé, D., 2003. Noise investigation of a high subsonic, moderate reynolds number jet using a compressible large eddy simulation. Theor. Comp. Fluid Dyn. 16 (4), 273-297.

Cambier, L., Heib, S., Plot, S., 2013. The Onera elsA CFD software: input from research and feedback from industry. Mech. Industr. 14 (03), 159-174.

Dairay, T., Fortuné, V., Lamballais, E., Brizzi, L.-E., 2015. Direct numerical simulation of a turbulent jet impinging on a heated wall. J. Fluid Mech. 764, 362-394.

Dewan, A., Dutta, R., Srinivasan, B., 2012. Recent trends in computation of turbulent jetimpingement impingement heat transfer. Heat Transfer Eng. 33 (4-5), 447-460.

Didden, N., Ho, C.-M., 1985. Unsteady separation in a boundary layer produced by an impinging jet. J. Fluid Mech. 160 (-1), 235.

Fosso, A., Deniau, H., Sicot, F., Sagaut, P., 2010. Curvilinear finite-volume schemes using high-order compact interpolation. J. Comput. Phys. 229 (13), 5090-5122.

Gardon, R., Akfirat, J.C., 1965. The role of turbulence in determining the heat-transfer characteristics of impinging jets. Int. J. Heat Mass Tran. 8 (10), 1261-1272.

Grenson, P., Léon, O., Reulet, P., Aupoix, B., 2016. Investigation of an impinging heated jet for a small nozzle-to-plate distance and high Reynolds number. Int. J. Heat Mass Trans. 102, 801-815.

Guerra, D.R.S., Su, J., Silva Freire, A.P., 2005. The near wall behavior of an impinging jet. Int. J. Heat Mass Trans. 48 (14), 2829-2840.

Hadžiabdić, M., Hanjalić, K., 2008. Vortical structures and heat transfer in a round impinging jet. J. Fluid Mech. 596, 221-260.

Hall, J.W., Ewing, D., 2006. On the dynamics of the large-scale structures in round impinging jets. J. Fluid Mech. 555, 439-458.

Han, B., Goldstein, R.J., 2001. Jet-impingement heat transfer in gas turbine systems. Ann. N. Y. Acad. Sci. 934 (1), 147-161.

Hattori, H., Nagano, Y., 2004. Direct numerical simulation of turbulent heat transfer in plane impinging jet. Int. J. Heat Fluid Fl. 25 (5), 749-758.

Lee, J., Lee, S., 1999. Stagnation region heat transfer of a turbulent axisymmetric jet impingement. Exp. Heat Transfer 12 (2), 137-156.

Lodato, G., Vervisch, L., Domingo, P., 2009. A compressible wall-adapting similarity mixed model for large-eddy simulation of the impinging round jet. Phys. Fluids 21 (3), 035102.

Natarajan, T., Jewkes, J.W., Lucey, D., Narayanaswamy, R., Chung, Y.M., 2016. Largeeddy simulations of a turbulent jet impinging on a vibrating heated wall. Int. J. Heat Fluid Fl.

Nicoud, F., Ducros, F., 1999. Subgrid-scale stress modelling based on the square of the velocity gradient tensor. Flow, Turbul. Combust. 62 (3), 183-200.

Piomelli, U., Chasnov, J.R., 1996. Large-eddy simulations: theory and applications. Turb. Trans. Model. (2), 269-336.

Popiel, C.O., Trass, O., 1991. Visualization of a free and impinging round jet. Exp. Therm. Fluid Sci. 4, 253-264.

Sagaut, P., Deck, S., Terracol, M., 2013. Multiscale and multiresolution approaches in turbulence: LES, DES and hybrid RANS/LES methods: applications and guidelines. World Scientific.

Tam, C., Dong, Z., 1996. Radiation and outflow boundary conditions for direct computation of acoustic and flow disturbances in a nonuniform mean flow. J. Comput. Acoust. 04 (02), 175-201.

Uddin, N., Neumann, S., Weigand, B., 2013. LES simulations of an impinging jet. Int. J. Heat Mass Trans. 57 (1), 356-368.

Visbal, M.R., Gaitonde, D.V., 2002. On the use of higher-order finite-difference schemes on curvilinear and deforming meshes. J. Comput. Phys. 181 (1), 155-185.

Zuckerman, N., Lior, N., 2006. Jet impingement heat transfer: physics, correlations, and numerical modeling. Adv. Heat Transfer 39, 565-631. 